\title{
Acylation of the Incretin Peptide Exendin-4 Directly Impacts Glucagon-Like Peptide-1 Receptor Signaling and Trafficking\$
}

\author{
Maria Lucey, Tanyel Ashik, Amaara Marzook, Yifan Wang, Joëlle Goulding, Atsuro Oishi, \\ Johannes Broichhagen, David J. Hodson, James Minnion, Yuval Elani, Ralf Jockers, \\ Stephen J. Briddon, Stephen R. Bloom, Alejandra Tomas, ${ }^{1}$ and Ben Jones ${ }^{1}$
}

\begin{abstract}
Section of Endocrinology and Investigative Medicine (M.L., T.A., A.M., J.M., S.R.B., B.J.) and Section of Cell Biology and Functional Genomics (Y.W., A.T.), Department of Metabolism, Digestion and Reproduction, and Department of Chemical Engineering (Y.E.), Imperial College London, London, United Kingdom; Division of Physiology, Pharmacology and Neuroscience, School of Life Sciences, University of Nottingham, Nottingham, United Kingdom (J.G., S.J.B.); Centre of Membrane Proteins and Receptors (COMPARE), University of Birmingham and University of Nottingham, Midlands, United Kingdom (J.G., D.J.H., S.J.B.); Université de Paris, Institut Cochin, INSERM, CNRS, Paris, France (A.O., R.J.); Department of Anatomy, Kyorin University Faculty of Medicine, Tokyo, Japan (A.O.); Leibniz-Forschungsinstitut für Molekulare Pharmakologie, Berlin, Germany (J.B.); Institute of Metabolism and Systems Research (IMSR), University of Birmingham, Birmingham, United Kingdom (D.J.H.); and Centre for Endocrinology, Diabetes and Metabolism, Birmingham Health Partners, Birmingham, United Kingdom (D.J.H.)
\end{abstract}

Received March 4, 2021; accepted July 14, 2021

\section{ABSTRACT}

The glucagon-like peptide-1 receptor (GLP-1R) is a class $B$ G protein-coupled receptor and mainstay therapeutic target for the treatment of type 2 diabetes and obesity. Recent reports have highlighted how biased agonism at the GLP-1R affects sustained glucose-stimulated insulin secretion through avoidance of desensitization and downregulation. A number of GLP-1R agonists (GLP-1RAs) feature a fatty acid moiety to prolong their pharmacokinetics via increased albumin binding, but the potential for these chemical changes to influence GLP-1R function has rarely been investigated beyond potency assessments for cAMP. Here, we directly compare the prototypical GLP-1RA exendin-4 with its Cterminally acylated analog, exendin-4-C16. We examine relative propensities of each ligand to recruit and activate $G$ proteins and $\beta$-arrestins, endocytic and postendocytic trafficking profiles, and interactions with model and cellular membranes in HEK293 and HEK293T cells. Both ligands had similar cAMP potency, but exendin-4-C16 showed $\sim 2.5$-fold bias toward G protein recruitment and a $\sim 60 \%$ reduction in $\beta$-arrestin-2 recruitment efficacy compared with exendin-4, as well as reduced GLP-1R endocytosis and preferential targeting toward recycling pathways. These effects were associated with reduced movement of the GLP-1R extracellular domain measured using a conformational biosensor approach and a $\sim 70 \%$ increase in insulin secretion in INS-1 832/3 cells. Interactions with plasma membrane lipids were enhanced by the acyl chain. Exendin-4-C16 showed extensive albumin binding and was highly effective for lowering of blood glucose in mice over at least 72 hours. Our study highlights the importance of a broad approach to the evaluation of GLP-1RA pharmacology.

\section{SIGNIFICANCE STATEMENT}

Acylation is a common strategy to enhance the pharmacokinetics of peptide-based drugs. This work shows how acylation can also affect various other pharmacological parameters, including biased agonism, receptor trafficking, and interactions with the plasma membrane, which may be therapeutically important.
A version of this article has been submitted to the preprint server bioRxiv (https://www.biorxiv.org/).

The Section of Endocrinology and Investigative Medicine is funded by grants from the Medical Research Council, Biotechnology and Biological Sciences Research Council National Institutes of Health Research (NIHR) and is supported by the NIHR Biomedical Research Centre Funding Scheme. The views expressed are those of the authors and not necessarily those of the funders. This project was also supported by the Medical Research Council [MR/R010676/1, MR/N00275X/1, and MR/S025618/1 and MR/N020081/1], the Imperial Post-doctoral Post-CCT Research Fellowship scheme, the European Federation for the Study of Diabetes, Diabetes UK [including Grant 17/0005681], the Academy of Medical Sciences, Society for Endocrinology, The British Society for Neuroendocrinology, the Engineering and Physical Sciences Research Council, the European Research Council under the European Union's Horizon 2020 research and innovation programme [Starting Grant 715884], UKRI Future Leaders Fellowship [MR/S031537/1], Fondation de la Recherche Médicale [Equipe FRM DEQ20130326503, EQU201903008055], Agence Nationale de la Recherche [ANR-19-CE16-0025-01, ANR-15-CE14-0025-02], Institut National de la Santé et de la Recherche Médicale, and Centre National de la Recherche Scientifique.

${ }^{1}$ A.T. and B.J. are co-senior authors.

dx.doi.org/10.1124/molpharm.121.000270.

S This article has supplemental material available at molpharm. aspetjournals.org.

\section{Introduction}

Type 2 diabetes (T2D) affects over 400 million people worldwide and leads to chronic illnesses including blindness, nerve damage, kidney failure, and cardiac disease (Guariguata et al., 2014). Pathologic increases in blood glucose, the central metabolic defect in T2D, occur chiefly because of failure of pancreatic $\beta$ cells to release enough of the glucoregulatory hormone insulin and overweight-related tissue resistance to insulin action. Therefore, pharmacological targeting of the glucagon-like peptide-1 receptor (GLP-1R), a class B G protein-coupled receptor (GPCR) that increases insulin release in a glucose-dependent manner and also suppresses appetite, leading to weight loss, is an effective strategy against T2D (Graaf et al., 2016). As GLP-1(7-36) $\mathrm{NH}_{2}$, the endogenous ligand for GLP-1R, is enzymatically inactivated in the circulation within minutes and eliminated by glomerular filtration, a number of 
strategies have been used to develop pharmaceutically viable peptide GLP-1R agonists (GLP-1RAs) that remain active in the circulation for hours or days (Andersen et al., 2018). As typified by exendin-4 (in clinical use as exenatide), the peptide amino acid sequence can be altered to prevent proteolytic degradation, extending circulatory half-life from minutes to hours while retaining full activity at the receptor (Kolterman et al., 2003). An alternative or complementary approach, the best known exemplars of which are liraglutide and semaglutide (Lau et al., 2015), involves attachment of a fatty acid side chain to the peptide to allow it to bind reversibly to albumin. Specifically, liraglutide includes a palmitic (C16) acid chain, which was switched to a C18 fatty diacid chain in semaglutide to improve affinity for human albumin (Knudsen and Lau, 2019). As albumin exceeds the size limit for glomerular filtration, this major elimination route is lost (Malm-Erjefält et al., 2010). Half-life protraction of semaglutide via this strategy allows once-weekly administration in humans (Knudsen and Lau, 2019).

Although GLP-1RA pharmacology has been traditionally assessed by measuring cAMP responses, additional factors are now recognized as important determinants of insulin release. For example, changes to the amino acid sequence of GLP-1R peptide agonists in their receptor core-facing N-terminal region can lead to bias between $\mathrm{G}$ protein signaling, $\beta$-arrestin recruitment, and receptor endocytosis (Zhang et al., 2015; Jones et al., 2018). We recently reported how a dual strategy based on both pharmacokinetically preferential acylation and $\mathrm{G}$ protein-directed biased agonism could optimize GLP-1R therapeutic efficacy (Lucey et al., 2020). Although not explored in detail in the latter project, we observed that the addition of a C-terminal acyl chain further influenced the ability of each ligand to recruit $\mathrm{G}$ proteins and $\beta$-arrestins to the GLP-1R, highlighting this feature as a modifier of GLP-1R efficacy in its own right.

Moreover, it is conceivable that acylation could constructively influence the interaction between agonist and plasma membrane by conferring distinct physicochemical properties, as suggested for the acylated analogs of GLP-2 (Trier et al., 2014). This has the potential to modulate downstream signaling by directing the ligand toward GLP-1Rs situated in submicroscopic membrane domains variably enriched with signaling effectors on the cytoplasmic side (Villar et al., 2016). Indeed, differences in segregation of activated GLP1Rs into these "membrane rafts" are an important feature of biased agonist action (Buenaventura et al., 2019).

To investigate these possibilities, in this study we performed a detailed comparison of the prototypical GLP-1R therapeutic agonist exendin- 4 with an equivalent peptide bearing a $\mathrm{C} 16$ fatty diacid at its $\mathrm{C}$ terminus, focusing on the relative propensities of each ligand to engage with $\mathrm{G}$ protein and $\beta$-arrestin recruitment and activation, endocytosis, and postendocytic sorting in HEK293 and HEK293T cells. We also evaluated interactions made by each ligand with giant unilamellar vesicles (GUVs) and the relative impact of modulating cellular cholesterol levels on GLP-1R signaling with each ligand. Our study highlights how installation of an acyl chain at the $\mathrm{C}$ terminus influences multiple pharmacological properties of exendin-4, enhancing its ability to stimulate insulin release from pancreatic $\beta$ cells.

\section{Materials and Methods}

Peptides and Reagents. All peptides were obtained from Wuxi Apptec (Wuhan, China) and were at least 90\% pure. Laboratory reagents were obtained from Sigma Aldrich (Dorset, UK) unless otherwise specified.

Cell Culture. HEK293T cells were maintained in Dulbecco's modified Eagle's medium (DMEM) supplemented with 10\% FBS and $1 \%$ penicillin/streptomycin. HEK293-SNAP-GLP-1R cells generated by stable transfection of pSNAP-GLP-1R (Cisbio, Paris, France) into HEK293 cells (Buenaventura et al., 2019) were used for some experiments and maintained in DMEM supplemented with $10 \%$ FBS, $1 \%$ penicillin/streptomycin, and $1 \mathrm{mg} / \mathrm{ml}$ G418. T-REx-SNAP-GLP-1R cells (Fang et al., 2020a), generated from parental Flp-In T-REx 293 cells (Thermo Fisher Scientific, MA) by Flp-mediated genomic integration of an insert encoding human GLP-1R with an N-terminal

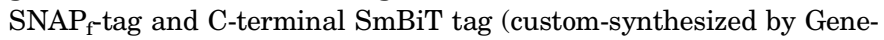
wiz, Steißlingen, Germany), with pcDNA5/FRT as the backbone, were maintained in DMEM supplemented with 10\% FBS and 1\% penicillin/streptomycin. SNAP-GLP-1R expression was induced using $0.1 \mu \mathrm{g} / \mathrm{ml}$ tetracycline for 24 hours prior to experiments. PathHunter GLP-1R $\beta$-arrestin-1 and -2 cells (DiscoverX) were maintained in Ham's F12 medium with $10 \%$ FBS and $1 \%$ penicillin/streptomycin. INS-1 832/3 cells (a gift from Prof. Christopher Newgard, Duke University) (Hohmeier et al., 2000) were maintained in RPMI at $11 \mathrm{mM}$ glucose, supplemented with $10 \%$ FBS, $10 \mathrm{mM}$ HEPES, $1 \mathrm{mM}$ pyruvate, $1 \mathrm{mM}$ pyruvate, $50 \mu \mathrm{M} \beta$-mercaptoethanol, and $1 \%$ penicillin/ streptomycin.

Equilibrium GLP-1R Binding Assays. HEK293-SNAP-GLP$1 \mathrm{R}$ cells were labeled using $40 \mathrm{nM}$ SNAP-Lumi4-Tb (Cisbio) in complete medium for 60 minutes at room temperature. After washing, cells were resuspended in Hanks' balanced salt solution (HBSS) containing $0.1 \%$ BSA and metabolic inhibitors (10 $\mathrm{mM} \mathrm{NaN}_{3}$ and $20 \mathrm{mM} \mathrm{2-deoxyglucose)} \mathrm{to} \mathrm{prevent} \mathrm{endocyto-}$ sis (Widmann et al., 1995). After 20 minutes at room temperature, cells in 96-well white plates were equilibrated to $4^{\circ} \mathrm{C}$ before addition of agonists. To measure binding parameters for exendin-4 and exendin-4-C16, these nonlabeled ligands were added alongside $10 \mathrm{nM}$ exendin(9-39)-FITC and incubated for 24 hours in the dark to allow binding equilibrium to be achieved. TR-FRET signal from each well was then recorded using a Flexstation 3 with the following settings: $\lambda_{\text {ex }} 340 \mathrm{~nm}, \lambda_{\mathrm{em}} 520$ and $620 \mathrm{~nm}$, autocutoff, delay $50 \mu \mathrm{s}$, integration time $300 \mu \mathrm{s}$. A saturation binding isotherm for exendin(9-39)-FITC was performed during each experiment, and the $\mathrm{K}_{\mathrm{i}}$ values for competing agonists were determined using the "Fit $\mathrm{K}_{\mathrm{i}}$ " preset of Prism 8.0. To measure saturation binding for FITC- or TMR-labeled exendin-4 and exendin-4-C16 directly, these ligands were added to cells and incubated as above, followed by measurement of TR-FRET signal using the following settings: FITC ligands: $\lambda_{\text {ex }} 340 \mathrm{~nm}$, $\lambda_{\mathrm{em}} 520$ and $620 \mathrm{~nm}$, autocutoff, delay $50 \mu \mathrm{s}$, integration time $300 \mu \mathrm{s}$; TMR ligands: $\lambda_{\mathrm{ex}} 340 \mathrm{~nm}, \lambda_{\mathrm{em}} 550$ and $610 \mathrm{~nm}$, autocutoff, delay $50 \mu \mathrm{s}$, integration time $300 \mu \mathrm{s}$.

\footnotetext{
ABBREVIATIONS: 3D, 3 dimensional; AUC, area under curve; BRET, bioluminescence resonance energy transfer; BSA, bovine serum albumin; CyOFP, cyan-excitable orange fluorescent protein; DERET, diffusion-enhanced resonance energy transfer; DMEM, Dulbecco's modified Eagle's medium; ECD, extracellular domain; FITC, fluorescein isothiocyanate; FRET, forster resonance energy transfer; GLP, glucagon-like peptide; GLP-1R, glucagon-like peptide-1 receptor; GLP-1RA, glucagon-like peptide-1 receptor agonist; GPCR, G protein-coupled receptor; GUV, giant unilameller vesicle; HBSS, Hanks' balanced salt solution; HTRF, homogeneous time-resolved fluorescence; IPGTT, intraperitoneal glucose tolerance test; $\mathrm{M} \beta \mathrm{CD}$, methyl- $\beta$-cyclodextrin; NA, numerical apperture; Nb37, nanobody-37; $\mathrm{PCH}$, photon counting histogram; Tb, terbium; T2D, type 2 diabetes; TMR, tetramethylrhodamine; TR-FRET, time-resolved Förster resonance energy transfer.
} 
Measurement of cAMP Production. HEK293-SNAP-GLP-1R cells were resuspended in serum-free medium and treated at $37^{\circ} \mathrm{C}$ with indicated concentration of agonist for 30 minutes. cAMP was then assayed by HTRF (Cisbio cAMP Dynamic 2) using a Spectramax i3x plate reader (Molecular Devices). Where indicated, cells were preincubated with methyl- $\beta$-cyclodextrin in advance of the assay before washing.

NanoBiT Assay. The assay was performed as previously described (Lucey et al., 2020). HEK293T cells in 12-well plates were transfected with $0.5 \mu \mathrm{g}$ GLP-1R-SmBiT plus $0.5 \mu \mathrm{g}$ LgBiT-mini-G (Wan et al., 2018) (a gift from Prof. Nevin Lambert, Medical College of Georgia), or with $0.05 \mu \mathrm{g}$ GLP-1R-SmBiT and $0.05 \mu \mathrm{g}$ LgBit$\beta$-arrestin-2 (Promega, Hampshire, UK) plus $0.9 \mu \mathrm{g}$ pcDNA3.1 for 24 hours. Cells were detached with EDTA and resuspended in HBSS, and furimazine (Promega) was added at a 1:50 dilution from the manufacturer's preprepared stock. After dispensing into 96-well white plates, a baseline read of luminescent signal was serially recorded over 5 minutes using a Flexstation 3 instrument (Molecular Devices) at $37^{\circ} \mathrm{C}$ before addition of the indicated concentration of ligand, after which the signal was repeatedly recorded for 30 minutes. Results were expressed relative to individual well baseline, and AUC was calculated. Each assay was performed with both pathways measured in parallel using the same ligand stocks. Bias between mini- $\mathrm{G}_{\mathrm{s}}$ and $\beta$-arrestin-2 recruitment was determined similarly to earlier work (Pickford et al., 2020), by subtracting the $\log \tau / \mathrm{K}_{\mathrm{A}}$ values (Kenakin et al., 2012) for each ligand in each pathway for each assay to determine $\Delta \log \tau / \mathrm{K}_{\mathrm{A}}$ (referred to here as $\Delta \log$ $\mathrm{R}$ ); this indicates the relative pathway preference for each ligand, which can then be compared statistically.

$\beta$-Arrestin-1 and -2 Recruitment by Enzyme Complementation. PathHunter GLP-1R $\beta$-arrestin- 1 or -2 cells were treated in serum-free medium for 30 minutes with a range of agonist concentrations before addition of PathHunter chemiluminescent detection reagents. Bias was assessed as in the section NanoBiT Assay.

$\boldsymbol{\beta}$-Arrestin Conformational Change Assay. The assay was adapted from a previous description (Oishi et al., 2019). HEK293T cells were seeded in six-well plates and transfected with $0.5 \mu \mathrm{g}$ NLuc- $\beta$-arrestin-2-CyOFP under a TK promoter, $0.5 \mu \mathrm{g}$ SNAP-GLP$1 \mathrm{R}$ under a CMV promoter, plus $1 \mu \mathrm{g}$ pcDNA3.1, for 24 hours before the assay. Alternatively, stable HEK293-SNAP-GLP-1R cells in sixwell plates were transfected with $0.5 \mu \mathrm{g}$ NLuc- $\beta$-arrestin-2-CyOFP plus $1.5 \mu \mathrm{g}$ pcDNA3.1. Cells were detached with EDTA and resuspended in HBSS with furimazine (1:50 dilution). After dispensing into 96 -well white plates, a baseline read of luminescent signals at both $460 \mathrm{~nm}$ and $575 \mathrm{~nm}$ was serially recorded over 5 minutes using a Flexstation 3 instrument at $37^{\circ} \mathrm{C}$. Ligands in HBSS were then added, after which signal was repeatedly recorded for 30 minutes. Results were first normalized to individual well baseline and then to the average vehicle signal within each assay (see Supplemental Fig. 5A). Statistical comparisons were performed on AUC calculated from each ligand-induced kinetic trace.

G Protein Activation Assay by Nb37 BRET. A construct encoding SNAP-GLP-1R with a C-terminal nanoluciferase was generated in-house by PCR cloning of the nanoluciferase sequence from pcDNA3.1-ccdB-Nanoluc (a gift from Mikko Taipale; Addgene plasmid \# 87067) onto the $\mathrm{C}$ terminus end of the SNAP-GLP-1R vector (Cisbio), followed by site-directed mutagenesis of the GLP-1R stop codon. HEK293T cells in six-well plates were transfected with $0.2 \mu \mathrm{g}$ SNAP-GLP-1R-NLuc, $0.2 \mu \mathrm{g}$ Nb37-GFP (a gift from Dr Roshanak Irranejad, UCSF), and $1.6 \mu \mathrm{g}$ pcDNA3.1 for 24 hours before the assay. Cells were detached with EDTA and resuspended in HBSS, and furimazine was added at a 1:50 dilution from the manufacturer's preprepared stock. After dispensing into 96 -well white plates, a baseline read of luminescent signals at both $460 \mathrm{~nm}$ and $525 \mathrm{~nm}$ was serially recorded over 5 minutes using a Flexstation 3 instrument at $37^{\circ} \mathrm{C}$. Ligands in HBSS were then added, after which signal was repeatedly recorded for 30 minutes. Results were first normalized to individual well baseline and then to the average vehicle signal from each assay (see Supplemental Fig. 5B). Statistical comparisons were performed on AUC calculated from each ligand-induced kinetic trace.

Preparation and Imaging of Fixed Cell Samples. Cells were seeded onto coverslips coated with $0.1 \%$ poly(D-lysine) and allowed to adhere overnight. Where relevant, transfection was performed as for functional assays, and surface labeling of SNAP-tagged GLP-1R was performed using $0.5 \mu \mathrm{M}$ of the indicated SNAP-Surface probe for 30 minutes at $37^{\circ} \mathrm{C}$ before washing with HBSS. Ligands were applied in Ham's F12 media containing $0.1 \%$ BSA at $37^{\circ} \mathrm{C}$. For fixation, $4 \%$ paraformaldehyde was applied directly to the medium for 15 minutes before washing with PBS. Slides were mounted in Prolong Diamond antifade with DAPI (Thermo Fisher Scientific) and allowed to set overnight. Imaging was performed using a Nikon Ti2E custom microscope platform with automated stage (ASI) and LED light source controlled by $\mu$ Manager. High-resolution $\mathrm{z}$-stacks were acquired via a $100 \times 1.45$ NA oil immersion objective and used for image deconvolution using DeconvolutionLab2 (Sage et al., 2017) with the Richardson-Lucy algorithm.

Measurement of GLP-1R Internalization by Time-Lapse High-Content Microscopy. HEK293-SNAP-GLP-1R cells were seeded overnight in black, clear-bottom 96-well imaging plates coated with $0.1 \%$ poly(D-lysine). Cells were labeled with SNAP-Surface-649 $(0.5 \mu \mathrm{M}$, from New England Biolabs, Hitchin, UK) for 30 minutes at $37^{\circ} \mathrm{C}$. After washing, imaging medium was added to the wells (phenol red-free DMEM with $10 \mathrm{mM}$ HEPES and $0.1 \%$ BSA) at $37^{\circ} \mathrm{C}$. Baseline epifluorescence and transmitted phase contrast images were acquired using a $20 \times$ magnification 0.75 NA objective from four positions per well using the imaging system in the section G Protein Activation Assay by Nb37 BRET. Further baseline epifluorescence images were acquired using a $40 \times$ magnification 0.95 NA objective, after which ligands were added directly to each well. Images in the same four positions were acquired every 3 minutes for 30 minutes. To quantify the translocation of surface-labeled SNAPGLP-1R from plasma membrane to endosomes, a "spot-counting" imaging-processing pipeline implemented in Fiji was developed as follows: 1) flatfield correction was applied to the epifluorescence images using BaSiC (Peng et al., 2017); 2) Laplacian filtering (smoothening scale: 2.0) was applied using FeatureJ; 3) starting with an image in which multiple endosomal puncta were visible, images were thresholded using the autoselected threshold from the "Triangle" algorithm, and the same threshold was used for all images; 4) spots were counted using the particle-counting algorithm in Fiji with a size limit of $0.5-5 \mu \mathrm{m}$ and roundness of $0.5-1$; 5) differences in cell density within each image were accounted for by estimating confluence from the phase contrast image (cropped to the region covered by the $40 \times$ objective) using PHANTAST (Jaccard et al., 2014) and dividing the spot count by this value; 6) after normalization, the number of spots present at baseline was subtracted to identify the ligand-induced changes. Rate constants were determined from one-phase exponential curve fitting in Prism 8.0.

Measurement of GLP-1R Internalization by DERET. The assay was performed as previously described (Lucey et al., 2020). HEK-SNAP-GLP-1R cells were labeled using $40 \mathrm{nM}$ SNAP-Lumi4$\mathrm{Tb}$ in complete medium for 60 minutes at room temperature. After washing, cells were resuspended in HBSS containing $24 \mu \mathrm{M}$ fluorescein and dispensed into 96 -well white plates. A baseline read was serially recorded over 5 minutes using a Flexstation 3 instrument at $37^{\circ} \mathrm{C}$ in TR-FRET mode with the following settings: $\lambda_{\mathrm{ex}} 340 \mathrm{~nm}, \lambda_{\mathrm{em}}$ 520 and $620 \mathrm{~nm}$, autocutoff, delay $400 \mu \mathrm{s}$, integration time $1500 \mu \mathrm{s}$. Ligands were then added, after which the signal was repeatedly recorded for 30 minutes. Fluorescence signals were expressed ratiometrically after first subtracting signal from wells containing $24 \mu \mathrm{M}$ fluorescein without cells. Internalization was quantified as AUC relative to individual well baseline.

Measurement of GLP-1R Trafficking to Acidic Endosomes Using LysoTracker. HEK-SNAP-GLP-1R cells were labeled with SNAP-Lumi4- $\mathrm{Tb}\left(40 \mathrm{nM}, 60\right.$ minutes at $37^{\circ} \mathrm{C}$, in complete medium), with LysoTracker Red DND99 (100 nM) added for the last 15 
minutes of the incubation period. After washing, labeled cells were resuspended in HBSS. TR-FRET signals at baseline and serially after agonist addition were recorded at $37^{\circ} \mathrm{C}$ using a Flexstation 3 plate reader using the following settings: $\lambda_{\mathrm{ex}}=335 \mathrm{~nm}, \lambda_{\mathrm{em}}=550$ and $610 \mathrm{~nm}$, delay $50 \mu \mathrm{s}$, integration time $300 \mu \mathrm{s}$. Receptor translocation to acidic endosomes was quantified as the ratio of fluorescent signal at $610 \mathrm{~nm}$ to that at $550 \mathrm{~nm}$.

Measurement of Receptor Recycling by TR-FRET. The assay was performed as previously described (Fang et al., 2020b). Adherent HEK293-SNAP-GLP-1R cells in 96-well white plates coated with $0.1 \%$ poly(D-lysine) were labeled with $40 \mathrm{nM}$ BG-S-S-Lumi4-Tb in complete medium for 60 minutes at room temperature. After washing, cells were treated for 30 minutes with each agonist at $100 \mathrm{nM}$ or vehicle (serum-free medium) to induce GLP-1R internalization. Cells were then washed once with HBSS before application of alkaline TNE buffer ( $\mathrm{pH}$ 8.6) containing (or not) $100 \mathrm{mM}$ Mesna for 5 minutes at $4^{\circ} \mathrm{C}$ to cleave residual surface GLP-1R. In total, $10 \mathrm{nM}$ Luxendin645 (Ast et al., 2020) in HBSS containing 0.1\% BSA was then added, and TR-FRET signal was serially monitored using a Spectramax i3x multimode plate reader with HTRF module. Ratiometric HTRF responses were normalized to baseline from the first three reads.

Measurement of GLP-1R Compartmentalization by BRET. HEK293T cells in six-well plates were transfected with $0.2 \mu \mathrm{g}$ SNAPGLP-1R-NLuc, $0.2 \mu \mathrm{g}$ of KRAS-Venus, Rab5-Venus, Rab7-Venus, or Rab11-Venus (kindly provided by Prof. Nevin Lambert, Medical College of Georgia, and Prof. Kevin Pfleger, University of Western Australia), plus $1.6 \mu \mathrm{g}$ pcDNA3.1 24 hours before the assay. Cells were detached with EDTA and resuspended in HBSS, and furimazine was added at a 1:50 dilution from the manufacturer's preprepared stock. After dispensing into 96 -well white plates, a baseline read of luminescent signals at both $460 \mathrm{~nm}$ and $535 \mathrm{~nm}$ was serially recorded over 5 minutes using a Flexstation 3 instrument at $37^{\circ} \mathrm{C}$. Ligands in HBSS were then added, after which signal was repeatedly recorded for 30 minutes. Results were first normalized to individual well baseline and then to the average vehicle signal from each experiment (see Supplemental Fig. 5, C-F). Statistical comparisons were performed on AUC calculated from each ligand-induced kinetic trace.

Mini-G $_{\mathbf{s}}$ Subcellular Translocation Assay. HEK293-SNAPGLP-1R cells were seeded in six-well plates and transfected with 0.2 $\mu \mathrm{g}$ plasmid DNA encoding mini- $\mathrm{G}_{\mathrm{s}}-\mathrm{NLuc}$ (a gift from Prof. Nevin Lambert, Medical College of Georgia) (Wan et al., 2018), $0.2 \mu \mathrm{g}$ KRAS-Venus or Rab5-Venus, plus $1.6 \mu \mathrm{g}$ pcDNA3.1 for 24 hours before the assay. Cells were detached with EDTA and resuspended in HBSS with furimazine (1:50 dilution). After dispensing into 96well white plates, a baseline read of luminescent signals at both 460 $\mathrm{nm}$ and $535 \mathrm{~nm}$ was serially recorded over 5 minutes using a Flexstation 3 instrument at $37^{\circ} \mathrm{C}$. Ligands in HBSS were then added, after which signal was repeatedly recorded for 30 minutes. Results were first normalized to individual well baseline and then to the average vehicle signal from each experiment (Supplemental Fig. 5, G and $\mathrm{H}$ ). Statistical comparisons were performed on AUC calculated from each ligand-induced kinetic trace.

Insulin Secretion Assay. INS-1 832/3 cells were preincubated in complete medium at $3 \mathrm{mM}$ glucose for 16 hours prior to the assay. After washing, cells were dispensed into 96-well plates containing 11 $\mathrm{mM}$ glucose \pm the indicated concentration of agonist and incubated for 1 hour (acute secretion assay) or 16-18 hours (sustained secretion assay). Secreted insulin was analyzed from a sample of diluted supernatant by immunoassay (Cisbio wide range HTRF assay, measured using a Spectramax i3x plate reader). Results were normalized to the glucose-only response.

Isolation and Imaging of Giant Unilamellar Vesicles. GUVs in the fluid phase, composed of 1-palmitoyl-2-oleoyl-sn-glycero-3-phosphocholine lipid were prepared via electroformation. Lipids were purchased from Avanti Polar Lipids. First, lipid in chloroform $(20 \mu \mathrm{l} ; 1 \mathrm{mg} / \mathrm{ml})$ was spread evenly on a conductive indium tin oxide-coated slide, leaving a film that was dried under vacuum for 30 minutes to remove residual solvent. A 5-mm-thick polydimethylsiloxane spacer with a central cutout was used to separate the slides with the conductive sides facing each other, and the chamber was filled with a solution of $100 \mathrm{mM}$ sucrose in DI water. An alternating electric field $(1 \mathrm{~V}, 10 \mathrm{~Hz})$ was applied across the indium tin oxide-coated plates using a function generator (Aim-TTi, TG315). After $2 \mathrm{~h}$, the electric field was changed to $1 \mathrm{~V}, 2 \mathrm{~Hz}$ for a further hour, and the resulting vesicles were collected. Either acylated or nonacylated compounds were added to make a final concentration of $100 \mathrm{nmol} / \mathrm{l}$, and the solution was incubated for 30 minutes. For imaging, $10 \mu \mathrm{l}$ of the vesicle suspension was added to $90 \mu \mathrm{l}$ of 100 $\mathrm{mM}$ glucose in DI water in an imaging chamber, and the vesicles sedimented on the bottom of the glass slide. Confocal imaging was done on a Leica TCS SP5 confocal fluorescent microscope with a $20 \times$ objective set with an $84.5-\mu \mathrm{m}$ pinhole (1 airy unit). Samples were acquired at a frequency of $400 \mathrm{~Hz}$ with eight line averages. The excitation was achieved at a wavelength of $495 \mathrm{~nm}$, with emission set at between 510 and $530 \mathrm{~nm}$. The images were acquired in the midplane of the GUVs. During data collection, focal planes slightly above and below were viewed to confirm image acquisition from the midplane.

Peptide-Membrane Interaction Assay by FRET. T-RExSNAP-GLP-1R cells were pretreated $\pm 0.1 \mu \mathrm{g} / \mathrm{ml}$ tetracycline to induce SNAP-GLP-1R expression. Cells were detached using EDTA and centrifuged, and the pellet was washed before the assay. Cellular labeling was then performed for 5 minutes using freshly prepared NR12S (Kucherak et al., 2010), a gift from Prof. Andrey Klymchenko, University of Strasbourg, at the indicated concentration in PBS in the dark. After washing three times to remove unbound probe, cells were dispensed into black 96-well microplates containing FITC-conjugated peptides at a final concentration of $100 \mathrm{nM}$. FRET measurements were taken using a Flexstation 3 instrument with the following settings: $\lambda_{\text {ex }} 475 \mathrm{~nm}, \lambda_{\text {em }}$ range $520-680 \mathrm{~nm}$ in 5 -nm increments, cutoff $495 \mathrm{~nm}$. Recorded fluorescence intensities were then normalized to $525 \mathrm{~nm}$ to allow for well-to-well differences and signal quenching by FRET. The normalized spectral trace from wells containing cells without NR12S probe was subtracted to determine probe-related FRET signal, which was quantified as AUC between 525 and $680 \mathrm{~nm}$. Gaussian fitting was performed to determine the peak emission wavelength and relative intensity.

GLP-1R Conformational Sensor Assay. HEK293-SNAP-GLP1R cells were labeled with Lumi4-Tb $\left(40 \mathrm{nM}, 60\right.$ minutes at $37^{\circ} \mathrm{C}$, in complete medium). After washing, cells were resuspended in HBSS containing NR12S (50 nM) and seeded into half-area opaque white plates. Baseline signal was measured for 5 minutes at $37^{\circ} \mathrm{C}$ using a Flexstation 3 plate reader with the following settings: $\lambda_{\mathrm{ex}}=335 \mathrm{~nm}$, $\lambda_{\mathrm{em}}=490$ and $590 \mathrm{~nm}$, delay $50 \mu \mathrm{s}$, integration time $300 \mu \mathrm{s}$. Ligands were then added, and signal was serially monitored for 10 minutes. The TR-FRET ratio, i.e., the ratio of fluorescence intensities at 590 and $490 \mathrm{~nm}$, was considered indicative of the proximity of the GLP$1 \mathrm{R}$ extracellular domain (ECD) to the plasma membrane.

Assessment of cAMP Signaling from Membrane Nanodomains by FRET. T-REx-SNAP-GLP-1R cells were transfected for 36 hours with $2 \mu \mathrm{g}$ per well of a six-well plate of the cAMP biosensor AKAP79-CUTie (a gift from Prof. Manuela Zaccolo, University of Oxford) (Surdo et al., 2017). At 6 hours after transfection, cells were transferred to black, clear-bottom imaging microplates in complete medium containing $0.1 \mu \mathrm{g} / \mathrm{ml}$ tetracycline to induce SNAP-GLP-1R expression over 24 hours. For imaging experiments, adherent cells in HBSS underwent FRET imaging by widefield microscopy using an image splitter (Cairn Optosplit III) to separate cyan fluorescent protein- and yellow fluorescence protein-wavelength emission light into separate images on the camera chip. Images were acquired every second for a 1-minute baseline period and then for 5 minutes after addition of a saturating $1 \mu \mathrm{M}$ agonist concentration. Channel registration was performed using the Cairn Image Splitter plug-in for ImageJ. Phase contrast images were used for segmentation, allowing quantification of mean fluorescence intensity from cell-containing and background fluorescence, with the latter being subtracted before calculation of cell-associated FRET ratio from each 
image. FRET signal was normalized to $t=0$ and then to the vehicle response. For plate reader experiments, FRET signal was monitored from wells before and up to 12 minutes after addition of a range of concentrations of each agonist using a Flexstation 3 plate reader at $37^{\circ} \mathrm{C}$ and the following settings: $\lambda_{\mathrm{ex}}=440 \mathrm{~nm}, \lambda_{\mathrm{em}}=485$ and $535 \mathrm{~nm}$. FRET was quantified as the ratio of fluorescent signal at $535 \mathrm{~nm}$ to that at $485 \mathrm{~nm}$ after subtraction of background signal at each wavelength. Cisbio lysis buffer was added immediately after completing the read, and total cAMP was determined by HTRF. Concentrationresponse curves were determined from average FRET signal from 8-12 minutes as well as the HTRF cAMP results to allow comparison of responses at an equivalent time point.

Fluorescence Correlation Spectroscopy. Fluorescence correlation spectroscopy was performed as previously described (Corriden et al., 2014) on a Zeiss LSM 510NLO ConfoCor 3 microscope fitted with a $40 \times$ c-Apochromat $1.2 \mathrm{NA}$ water-immersion objective. At $22^{\circ} \mathrm{C}, 30$-second reads were taken with a diode-pumped solid-state 561 -nm laser excitation at $\sim 0.2 \mathrm{kWcm}^{-2}$, emission was collected through a 580- to $610-\mathrm{nm}$ bandpass, and the pinhole was set to 1 airy unit. The measurement volume was calibrated on each experimental day with $20 \mathrm{nM}$ Rhodamine $6 \mathrm{G}$ (diffusion coefficient, D, $2.8 \times$ $10^{-10} \mathrm{~m}^{2} \mathrm{~s}^{-1}$ ) in high performance liquid chromatography-grade water. Exendin-4-TMR and Exendin-4-TMR-C16 were prepared in HBSS with or without $0.1 \%$ BSA in an eight-well Nunc Laboratorytek chambered coverglass (No. 1.0 borosilicate glass bottom) with the measurement volume placed $200 \mu \mathrm{m}$ above the coverslip bottom. Autocorrelation curves were modeled in Zen 2012 (Carl Zeiss, Jena) to describe a single 3D diffusing component under free diffusion (Corriden et al., 2014). To determine the average molecular brightness of the labeled peptide, measurement reads were reanalyzed by photon counting histogram (PCH) analysis within Zen 2012 (Huang et al., 2004). PCH analysis can quantify the average molecular brightness (photon counts per molecule) of a species and also provides a means to separate and quantify the concentration of two species that differ in their average molecular brightness. The laser beam profile was approximated to follow a 3D Gaussian distribution, measurement reads were binned at $20 \mu \mathrm{s}$, appropriate for fluorescent species in solution, and the first order correction was calculated daily following calibration with $20 \mathrm{nM}$ Rhodamine $6 \mathrm{G}$.

In Vivo Study. Animals were maintained in specific pathogen-free facilities, with ad libitum access to food (except prior to fasting studies) and water. Studies were regulated by the UK Animals (Scientific Procedures) Act 1986 of the UK and approved by Imperial College London (Project License PB7CFFE7A). Male C57Bl/6 mice (Charles River, UK) were fed a $60 \%$ high-fat diet (D12492; Research Diets) for 3 months prior to the study to induce obesity and glucose intolerance. The study began after an additional 1-week acclimatization period during which mice were singly housed and received sham intraperitoneal injections. Mice were randomly allocated to treatment, with average group weight confirmed to be similar postrandomization. The study began at the beginning of the dark phase with a single dose of each agonist or vehicle, combined with an intraperitoneal glucose tolerance test (IPGTT). Agonist was prepared in $20 \%$ glucose solution at a volume to provide the indicated weight-adjusted agonist dose and $2 \mathrm{~g} / \mathrm{kg}$ glucose. Blood glucose was monitored before and at 20-minute intervals after agonist/ vehicle/glucose administration using a handheld glucose meter. After 72 hours, the IPGTT was repeated at the beginning of the dark phase without further agonist administration. Food intake was assessed during the study by weighing of food at set intervals. Changes to body weight were also assessed at set intervals.

Experimental Design and Statistical Analysis Considerations. A preliminary finding that exendin-4-C16 shows reduced $\beta$-arrestin-2 recruitment compared with exendin-4 (Lucey et al., 2020) gave us a reasonable expectation that the two ligands would display alterations to other aspects of their pharmacology. Therefore, we adopted an approach in which we planned to perform at least five independent replicates for quantitative cell culture assays as recommended by some authorities (Curtis et al., 2018). We did not perform a formal power calculation. In some instances, we observed more subtle differences between compounds that required additional repeats to gain confidence in the magnitude of the effects size, which were then performed to allow comparisons between assays evaluating similar phenomena (e.g., BRET vs. TR-FRET assays). Animal experiments were performed with seven to eight mice per group without a formal power calculation but on the basis of our prior experience in determining meaningful differences between GLP-1RAs. The investigators were not fully blinded for in vivo studies but did not have reference to treatment allocation while conducting experiments; in vitro experiments were unblinded. No mice were excluded from the analyses. In cell culture experiments, technical replicates were averaged so that each individual experiment was treated as one biologic replicate. Quantitative data were analyzed using Prism 8.0 (GraphPad Software). Dose responses were analyzed using threeor four-parameter logistic fits, with constraints imposed as appropriate. Bias analyses were performed as described in the section NanoBiT Assay. Statistical comparisons were made by $t$ test or ANOVA as appropriate, with paired or matched designs used depending on the experimental design. Means \pm S.E.M., with individual replicates in some cases, are displayed throughout. Statistical significance was inferred if $P<0.05$, without ascribing additional levels of significance.

\section{Results}

Exendin-4-C16 Is a G Protein-Biased Ligand at GLP-1R. An exendin- 4 analog with a $\mathrm{C} 16$ diacid at the $\mathrm{C}$ terminus with a GK linker, originally described in our earlier publication (Lucey et al., 2020), was used in the present study and is referred to as "exendin-4-C16" (Fig. 1A). Competition binding experiments with FITC-conjugated antagonist ligand exendin(9-39) in HEK293 cells stably expressing SNAP-GLP1R (Fang et al., 2020b) indicated an approximately 2-fold reduction in binding affinity for exendin-4-C16 compared with unmodified exendin-4 (Fig. 1B; Table 1). A minor reduction in cAMP signaling potency that was not statistically significant was also observed (Fig. 1C; Table 1). To investigate the possibility that exendin-4-C16 may display altered preference for coupling to intracellular effectors, i.e., biased agonism, we used nanoluciferase complementation to measure recruitment of mini- $\mathrm{G}_{\mathrm{s}}$ (Wan et al., 2018) and $\beta$-arrestin-2 (Dixon et al., 2016) to the GLP-1R. This assay indicated that efficacy for $\beta$-arrestin-2 recruitment was particularly reduced with exendin-4-C16, with bias quantification using a standard approach (van der Westhuizen et al., 2014), confirming preferential coupling to mini- $\mathrm{G}_{\mathrm{s}}$ recruitment (Fig. 1D; Table 1). $\beta$-Arrestin-1 and $\beta$-arrestin-2 recruitment were also measured using the PathHunter assay, showing no evidence of divergence in isoform selectivity (Supplemental Fig. 1A).

As these complementation assays measure effector recruitment but not activation, we also performed further experiments to detect ligand-induced conformational changes in $G_{s}$ and $\beta$-arrestin-2. For the former, we recorded dynamic changes in intermolecular BRET signal between GLP-1R tagged at the $\mathrm{C}$ terminus with nanoluciferase and GFP-tagged $\mathrm{Nb37}$, a genetically encoded intrabody that recognizes and is recruited to active $\mathrm{G} \alpha_{\mathrm{s}}$ conformations (Irannejad et al., 2013). For the latter, we used an intramolecular $\beta$-arrestin-2 BRET sensor in which conformational changes lead to an increase in proximity between nanoluciferase at the $\mathrm{N}$ terminus and CyOFP at the $\mathrm{C}$ terminus (Oishi et al., 2019); this phenomenon is typically thought to indicate transition to an active state that could facilitate kinase activation (Nuber et al., 2016), although this was not specifically determined in our 
A

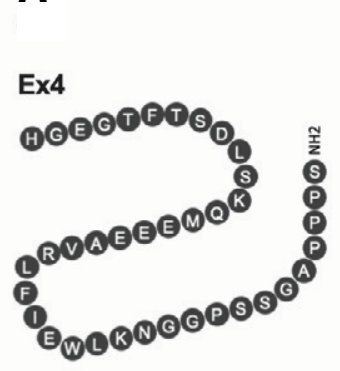

D Ex4

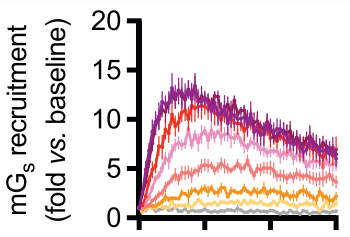

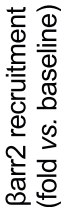

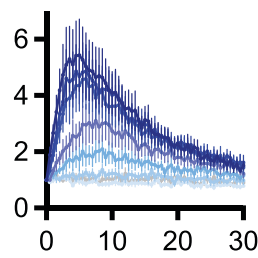

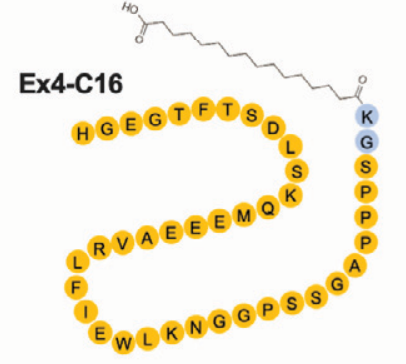

B
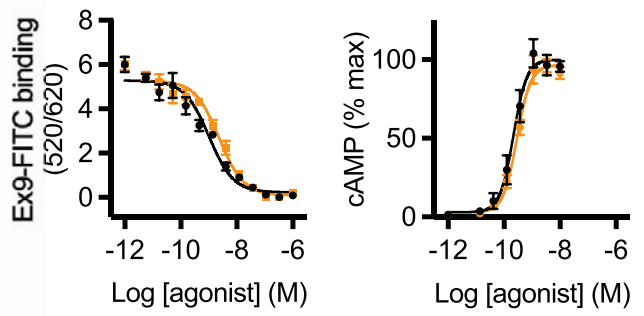

Ex4-C16 Log [agonist] (nM)
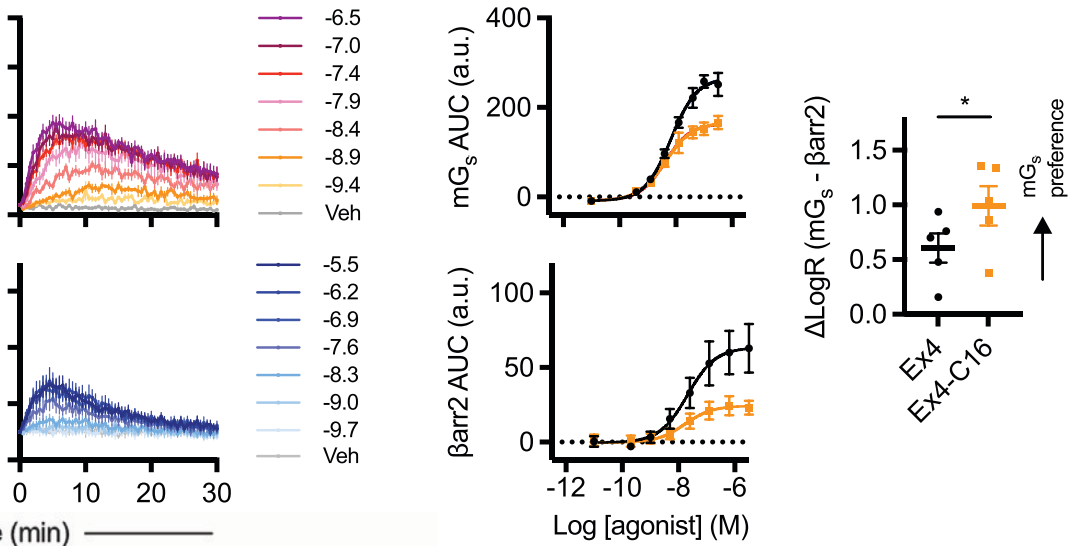

$F$

E

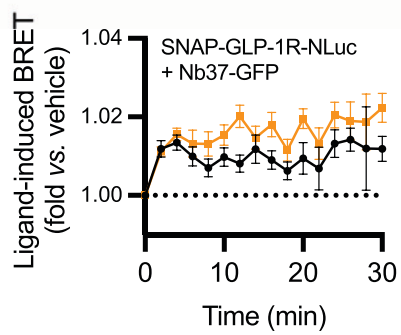

G

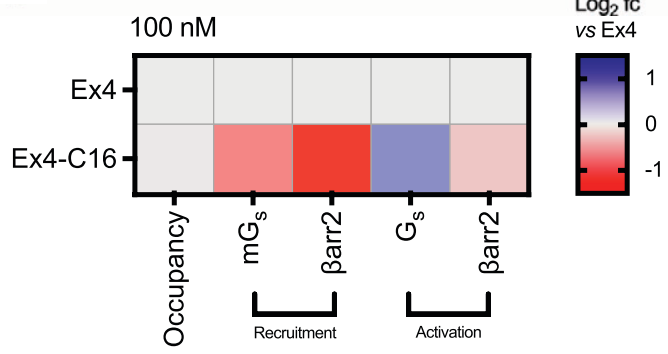

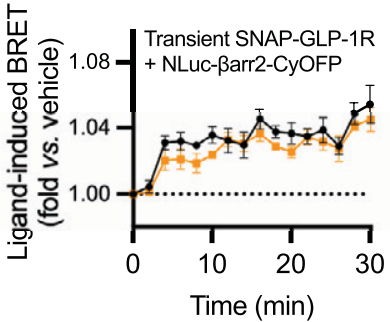

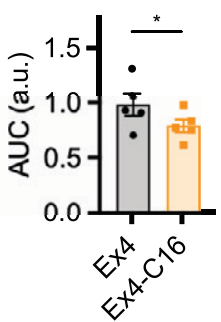

H

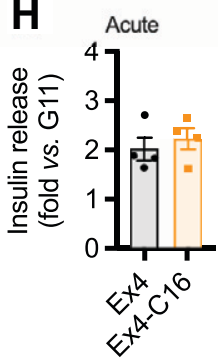

I Sustained

Log [agonist] (M)

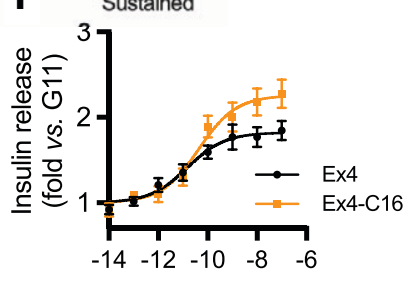

Fig. 1. Biased agonism with exendin-4 and exendin-4-C16. (A) Schematic depicting the amino acid sequences of exendin-4 and exendin-4-C16 in single-letter amino acid code. (B) Equilibrium binding of unmodified exendin-4 and exendin-4-C16, $n=5$, measured by competition binding in HEK293-SNAP-GLP1-R cells with exendin(9-39)-FITC used as the competing probe. (C) cAMP response in HEK293-SNAP-GLP-1R cells, 30minute stimulation, $n=5$, with four-parameter fits shown after normalization to global maximum. (D) LgBiT-mini-G $\left(\mathrm{mG}_{\mathrm{s}}\right)$ and $\mathrm{LgBit}_{\mathrm{s}}$ $\beta$-arrestin-2 ( $\beta$ arr2) recruitment to GLP-1R-SmBiT, $n=5$, with three-parameter concentration-responses constructed from AUCs and bias factor calculation (see Materials and Methods) with comparison by paired $t$ test. (E) Measurement of $\mathrm{G} \alpha_{\mathrm{s}}$ activation in HEK293T cells transiently transfected with SNAP-GLP-1R-NLuc and Nb37-GFP and stimulated with $100 \mathrm{nM}$ agonist or vehicle, $n=6$, with AUCs compared by paired $t$ test. Refer to Supplemental Fig. 5B for individual experimental repeats. (F) Measurement of $\beta$-arrestin-2 activation in HEK293T cells transiently transfected with SNAP-GLP-1R and NLuc-4myc- $\beta$ arr2-CYOFP1 and stimulated with $100 \mathrm{nM}$ agonist or vehicle, $n=5$, with AUCs compared by paired $t$ test. (G) Heatmap representation of $100 \mathrm{nM}$ agonist response data shown in Fig. 1, B-F with normalization to exendin-4 response. (H) Insulin secretion in INS-1 832/3 cells stimulated for 60 minutes with each agonist at $11 \mathrm{mM}$ glucose $(\mathrm{G} 11)$, $n=4$, paired $t$ test. (I) As for (H), but 16 -hour stimulation, $n=6$. Data are shown as means \pm S.E.M. with individual replicates shown in some cases. $* P<0.05$ by statistical test indicated. 
TABLE 1

Pharmacological parameters of exendin-4 and exendin-4-C16 Mean \pm S.E.M. parameter estimates from concentration-response analyses in Fig. 1.

\begin{tabular}{|c|c|c|}
\hline & Exendin-4 & Exendin-4-C16 \\
\hline \multicolumn{3}{|c|}{ Binding affinity (HEK293-SNAP-GLP-1R, $n=5$ ) } \\
\hline $\log K_{i}(M)$ & $-9.2 \pm 0.1$ & $-8.8 \pm 0.1^{*}$ \\
\hline \multicolumn{3}{|c|}{ Cyclic AMP (HEK293-SNAP-GLP-1R, $n=5$ ) } \\
\hline $\log \mathrm{EC}_{50}(\mathrm{M})$ & $-9.7 \pm 0.1$ & $-9.5 \pm 0.1$ \\
\hline $\mathrm{E}_{\max }(\%$ global max $)$ & $101 \pm 2$ & $98 \pm 3$ \\
\hline Hill slope & $2.1 \pm 0.3$ & $1.8 \pm 0.3$ \\
\hline \multicolumn{3}{|c|}{ Mini-G $\mathrm{G}_{\mathrm{s}}$ recruitment $(\mathrm{HEK} 293 \mathrm{~T}, n=5)$} \\
\hline $\log \mathrm{EC}_{50}(\mathrm{M})$ & $-8.2 \pm 0.1$ & $-8.3 \pm 0.1$ \\
\hline $\mathrm{E}_{\max }$ & $268 \pm 23$ & $171 \pm 14^{*}$ \\
\hline \multicolumn{3}{|c|}{$\beta$-arrestin-2 recruitment (HEK293T, $n=5)$} \\
\hline $\log \mathrm{EC}_{50}(\mathrm{M})$ & $-7.6 \pm 0.1$ & $-7.7 \pm 0.1$ \\
\hline $\mathrm{E}_{\max }(\mathrm{AUC})$ & $66 \pm 16$ & $25 \pm 5^{*}$ \\
\hline \multicolumn{3}{|c|}{ Insulin secretion (INS-1 832/3, $n=6$ ) } \\
\hline $\log \mathrm{EC}_{50}(\mathrm{M})$ & $-11.0 \pm 0.2$ & $-10.6 \pm 0.1^{*}$ \\
\hline $\mathrm{E}_{\max }$ & $1.7 \pm 0.1$ & $2.2 \pm 0.2^{*}$ \\
\hline
\end{tabular}

${ }^{*} P<0.05$ by paired $t$ test.

study. These studies suggested reduced $\beta$-arrestin- 2 conformational rearrangement with exendin-4-C16 but, interestingly, increased $\mathrm{G} \alpha_{\mathrm{s}}$ activation (Fig. 1, E and F; Supplemental Fig. $1 B$ ), which could be a consequence of reduced $\beta$-arrestinmediated desensitization. This $\mathrm{G} \alpha_{\mathrm{s}}$ activation assay showed a low dynamic range, but Ex4-C16 consistently showed a subtly increased response across all experimental repeats (Supplemental Fig. 5B). Note that the apparent increase in $\mathrm{G} \alpha_{\mathrm{s}}$ activation is still compatible with a lack of difference in measured $\mathrm{E}_{\text {max }}$ for cAMP (Fig. 1C), as adenylate cyclase activity may be saturated at submaximal activation of the cellular $\mathrm{G} \alpha_{\mathrm{s}}$ pool. Ligand pharmacology is summarized in the heatmap shown in Fig. 1G, indicating how, at a $100 \mathrm{nM}$ dose, to achieve close to $100 \%$ receptor occupancy, $\mathrm{G}_{\mathrm{s}}$ recruitment and activation are favored compared with $\beta$-arrestin-2 responses with exendin-4-C16.

As G protein-directed agonism is now established as a means to improve GLP-1R-mediated insulinotropic efficacy by avoiding receptor desensitization over prolonged stimulation periods (Jones et al., 2018; Fremaux et al., 2019; Fang et al., 2020b; Lucey et al., 2020), we also treated INS-1 832/3 clonal $\beta$ cells (Hohmeier et al., 2000) for both 1 and 16 hours with exendin- 4 and exendin-4-C16 and measured cumulative insulin secretion. As expected, there was no difference in acute insulin release (Fig. $1 \mathrm{H}$ ), but the maximum response was increased with the -C16 ligand (Fig. 1I; Table 1).

Exendin-4-C16 Triggers Slower GLP-1R Internalization Compared with Exendin-4. GLP-1R undergoes rapid agonist-induced internalization (Widmann et al., 1995), a process that is intrinsically linked to the spatial orchestration of intracellular signal generation (Fletcher et al., 2018; Tomas et al., 2019; Manchanda et al., 2021). Both exendin-4 and exendin-4-C16 resulted in extensive endocytosis of surface-labeled SNAP-GLP-1R (Fig. 2A). To quantify this process, we used high-content time-lapse microscopy in which the translocation of surface-labeled SNAP-GLP-1R into the endocytic network is determined from the appearance an intracellular punctate distribution of fluorescent signal (Fig. 2B; Supplemental Fig. 2A; Supplemental Video 1). Treatment with exendin-4 resulted in faster and more extensive appearance of fluorescent puncta at higher ligand concentrations.
Notably, the number of puncta tended to reduce at later time points with exendin- 4 because of the coalescence in a perinuclear location where multiple punctate endosomal structures which could no longer be individually resolved.

We also used tetramethylrhodamine (TMR)-tagged conjugates of each ligand, with the fluorophore installed at position K12, previously shown to be well tolerated by exendin-4 (Clardy et al., 2014; Jones et al., 2018; Pickford et al., 2020) and validated for GLP-1R binding by TR-FRET (Supplemental Fig. 2B) and cAMP measurements (Supplemental Fig. 2C), with the latter showing modest reductions in potency for both TMR conjugates. Using the tetracycline-inducible T-REx system to modulate SNAP-GLP-1R expression (Fang et al., 2020a), both fluorescent ligands were shown to bind specifically to the receptor and demonstrated extensive uptake into the endosomal compartment (Fig. 2C).

Endosomal Trafficking Differences between Exendin-4 and Exendin-4-C16. To gain insights into ligandinduced GLP-1R movements to or from different subcellular compartments, we used time-resolved proximity-based energy transfer techniques, with both chemical (for FRET) or genetically encoded (for BRET) acceptors as localization markers, providing complementary readouts. SNAP-GLP-1R labeled at the $\mathrm{N}$ terminus with the lanthanide probe Lumi4Tb can transfer energy to time-resolved FRET acceptors situated in the extracellular space or the endosomal lumen (Fig. 3A). DERET was used to quantify movement of GLP-1R away from the cell surface as a reduction in signal transfer to fluorescein-containing extracellular buffer (Levoye et al., 2015); this confirmed that GLP-1R internalization was slower for exendin-4-C16 (Fig. 3B; Supplemental Fig. 3A). We developed a further assay in which the trafficking of GLP-1R to late endosomes/lysosomes was detected by energy transfer to the lysomotropic fluorescent dye LysoTracker-DND99 (Fig. $3 \mathrm{C})$. This suggested that exendin-4-C16 treatment led to markedly less targeting of GLP-1R to this degradative compartment and, as this difference was more marked than for the internalization reading by DERET, that this reflects a difference in postendocytic targeting rather than simply less GLP-1R internalization. In contrast, postendocytic recycling of GLP-1R back to the plasma membrane, measured by time-resolved FRET (Fang et al., 2020b) between reemergent GLP-1R and Cy5-labeled antagonist analog of exendin(9-39) (Ast et al., 2020), was faster after treatment with exendin-4-C16 (Fig. 3D). TR-FRET data are also summarized and statistically compared in Fig. 3E.

Bystander BRET can be used to monitor the translocation of GPCRs within the endocytic network (Tiulpakov et al., 2016). We observed a ligand-induced reduction in BRET signal when SNAP-GLP-1R tagged at the $\mathrm{C}$ terminus with nanoluciferase was coexpressed with the plasma membrane marker KRASVenus; the effect was more pronounced with exendin-4 than with exendin-4-C16 (Fig. 3F). A concomitant increase in BRET signal to Rab5-Venus was seen, indicating entry of the receptor into early endosomes (Fig. 3G). The similar Rab5 BRET signal for each ligand in the face of apparently different GLP-1R internalization rates might be reconciled by the observation that, after exendin-4-C16 treatment, a subtly reduced signal from Rab7-positive late endosomes was detected (Fig. $3 \mathrm{H}$ ), whereas a greater signal was recorded from Rab11-positive recycling endosomes (Fig. 3I). This pattern is suggestive of preferential trafficking of GLP-1R toward a recycling pathway with exendin-4-C16 
A
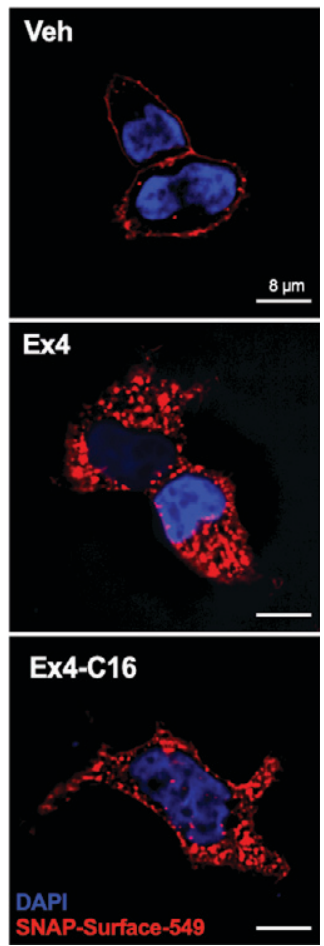

C
B
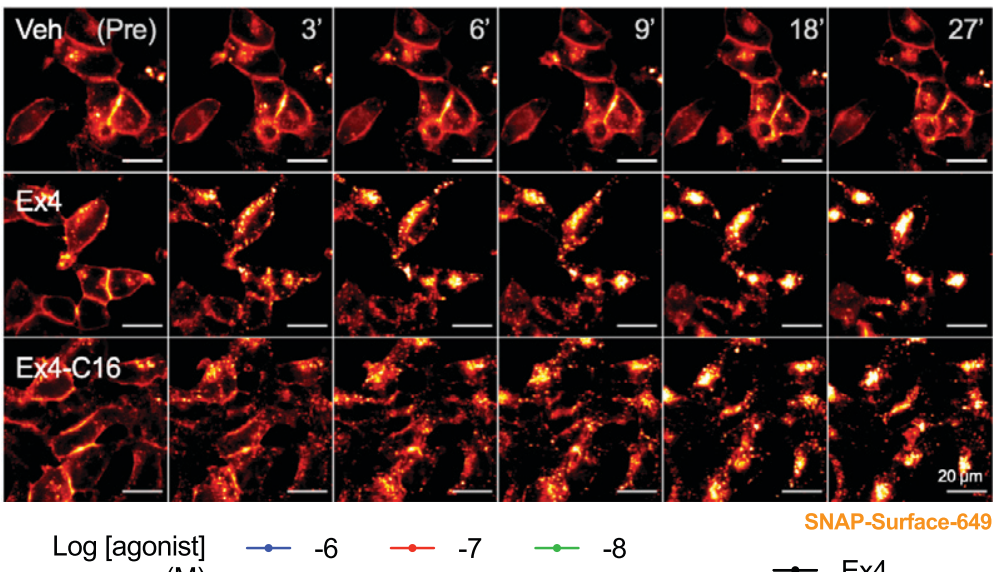

(M) $\rightarrow-9 \rightarrow-10 \multimap$ Veh
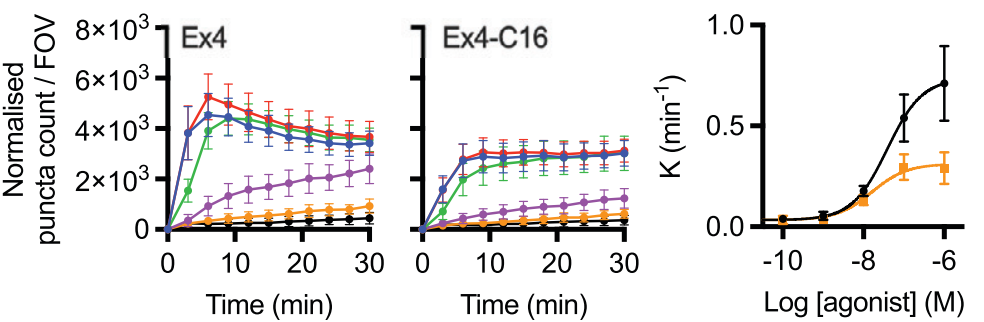
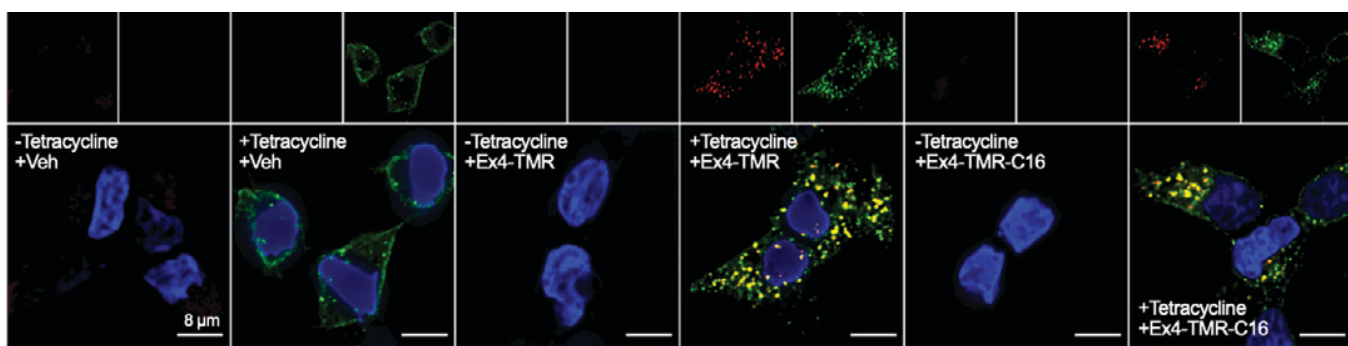

DAPI TMR SNAP-Surface-649

Fig. 2. Visualization of GLP-1R endocytosis with exendin-4 and exendin-4-C16, and fluorescent agonist conjugates. (A) Representative images from $n=3$ experiments demonstrating endocytosis of SNAP-GLP-1R labeled with SNAP-Surface-549 after treatment of 30 minutes with $100 \mathrm{nM}$ agonist or vehicle; scale bars, $8 \mu \mathrm{m}$. (B) Time-lapse images demonstrating movement of surface-labeled SNAP-GLP-1R into punctate structures on stimulation with $100 \mathrm{nM}$ agonist or vehicle; scale bars, $20 \mu \mathrm{m}$. The graphs show the change over time in "spot count" per $0.33-\mathrm{mm}$ field of view (FOV) after normalization to cell confluence, with the concentration-dependent rate constant (K) derived from one-phase association fitting also plotted with a three-parameter fit. (C) Representative images showing endosomal uptake of exendin-4 and exendin-4-TMR in T-REx-SNAP-GLP$1 \mathrm{R}$ cells with or without tetracycline-induced GLP-1R expression, labeled with SNAP-Surface-649 prior to stimulation with $100 \mathrm{nM}$ of each TMRconjugate for 30 minutes. Scale bars, $8 \mu \mathrm{m}$. Data are shown as means \pm S.E.M.

treatment, resulting in a reduced net rate of surface receptor loss. BRET results were broadly in accordance with results from the TR-FRET assays, although differences were generally smaller while remaining statistically significant (Fig. 3J).

GLP-1R cAMP signaling was reported to originate from early endosomes as well as the plasma membrane (Girada et al., 2017). As an indirect readout of this process, we monitored the redistribution of nanoluciferase-tagged mini- $\mathrm{G}_{\mathrm{s}}$ to different subcellular compartments after agonist stimulation. A rapid increase in mini- $\mathrm{G}_{\mathrm{s}}$-to-KRAS (plasma membrane) BRET signal was apparent with both ligands (Fig. 3K), but with a slightly higher peak response with exendin-4, consistent with the higher efficacy displayed by this ligand for $G$ protein recruitment (Fig. 1D). A decline in signal at later time points was observed with exendin-4 but not exendin-4-
C16, which may result from more extensive $\beta$-arrestin-mediated steric hinderance or internalization of GLP-1R with the former ligand. Interestingly, mini- $\mathrm{G}_{\mathrm{s}}$-to-Rab5 (early endosome) BRET signal amplitude was considerably reduced for exendin-4-C16 (Fig. 3K), despite the fact that the receptor localization in the Rab5 compartment was similar for both ligands (Fig. 3G).

These data indicate that C-terminal acylation alters the trafficking profile of exendin-4, favoring GLP-1R sorting toward a recycling rather than degradative pathway. The observation that mini- $\mathrm{G}_{\mathrm{s}}$ protein recruitment to early endosomes was reduced with exendin-4-C16, yet this ligand shows higher efficacy for sustained insulin secretion, suggests that signaling from early endosomes may not be a dominant mechanism for prolonged signaling with GLP-1R under 
A
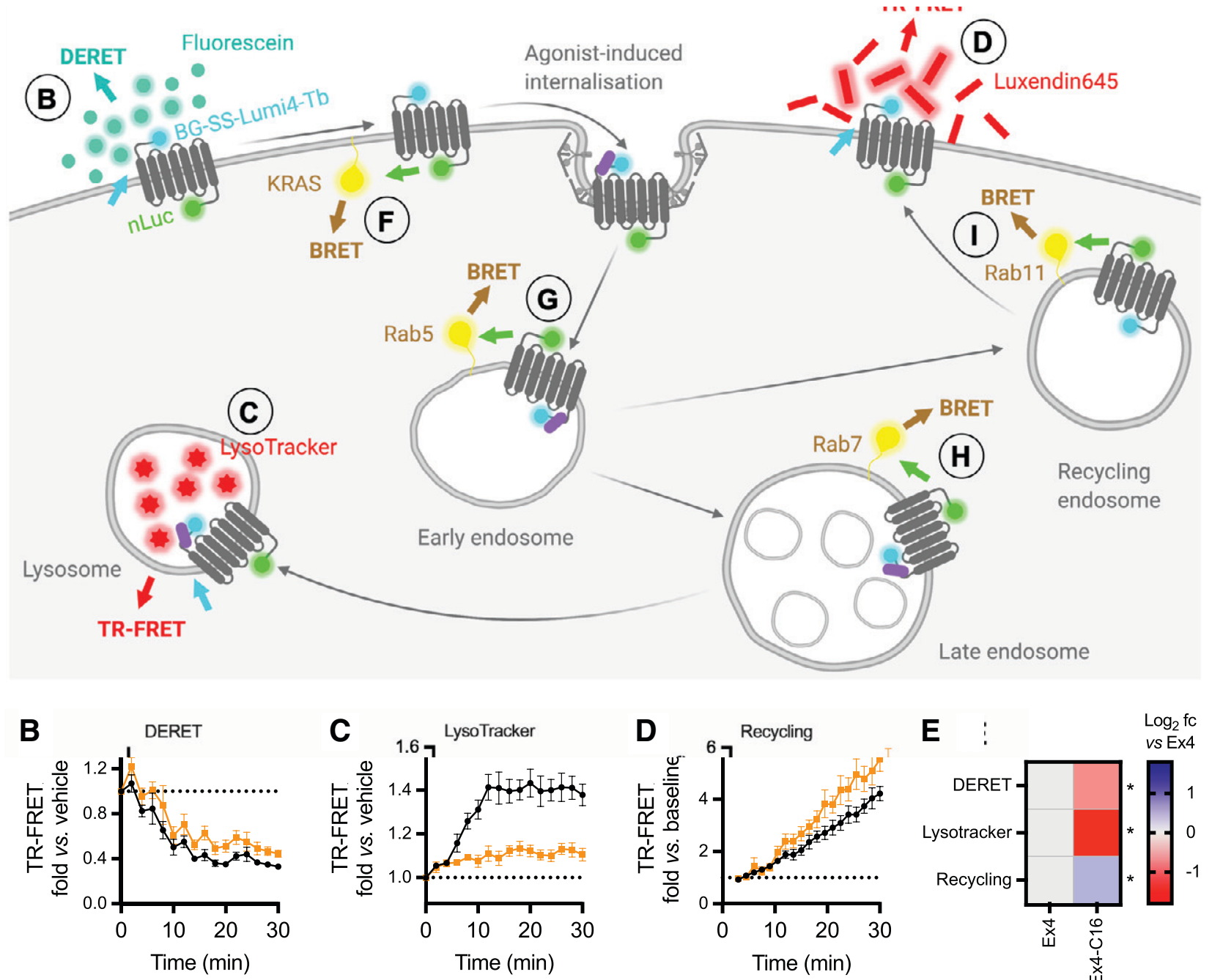

E :

$\log _{2} \mathrm{fc}$
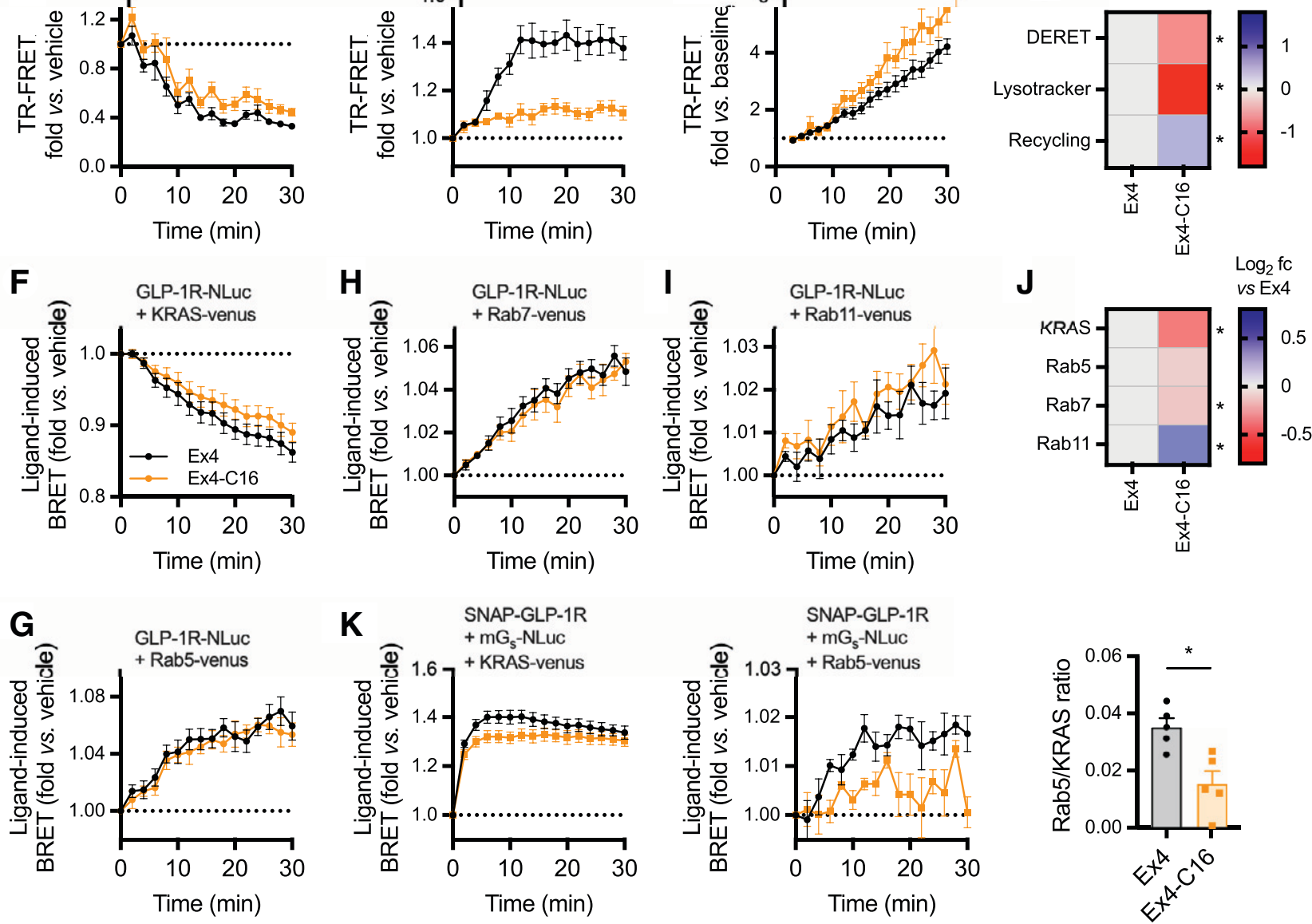

Fig. 3. Subcellular targeting of exendin-4 and exendin-4-C16. (A) Schematic depicting the investigation of GLP-1R membrane trafficking using the TR-FRET and BRET assays used in this work. (B) GLP-1R disappearance from cell surface in HEK293-SNAP-GLP-1R cells treated with 100 $\mathrm{nM}$ agonist or vehicle, measured by DERET, $n=5$. (C) GLP-1R translocation to late endosomes/lysosomes in HEK293-SNAP-GLP-1R cells treated with $100 \mathrm{nM}$ agonist or vehicle, measured by TR-FRET using LysoTracker Red DND99 as the acceptor, $n=5$. (D) GLP-1R recycling in HEK293- 
pharmacological conditions, at least with exendin-4 derived GLP-1R agonists.

Acylation Affects the Interaction of the Exendin-4 Peptide with the Plasma Membrane. Several studies report an interaction of GLP-1R agonist peptides with model membranes, typically resulting in enhanced stability of the helical secondary structure (Thornton and Gorenstein, 1994; Neidigh et al., 2001; Andersen et al., 2002; Fox et al., 2009). Acylation of glucagon-like peptide-2 increases its tendency to interact with lipid bilayers (Trier et al., 2014). Similarly, the C16 chain of exendin-4-C16 might promote selective engagement with membrane regions dependent on local hydrophobicity. We performed confocal microscopy of FITC conjugates of exendin-4 and exendin-4-C16 bound to GUVs, prepared via electroformation. Both FITC ligands were conjugated at position $\mathrm{K} 12$, as for the equivalent TMR conjugates, and retained GLP-1R binding properties (Supplemental Fig. 4A) and cAMP signaling (Supplemental Fig. 4B). When the acylated compound was present in the sample, clear fluorescence accumulation was seen on the membrane surface, in contrast to the nonacylated compound, in which no accumulation was seen (Fig. 4A). This demonstrates that the presence of the C16 acyl chain promotes insertion of the molecule into model lipid bilayers.

To establish whether this phenomenon is relevant to living cells, we measured FRET between FITC ligands and the plasma membrane of T-REx-SNAP-GLP-1R cells labeled with the solvatochromic probe NR12S (Kucherak et al., 2010). The excitation spectrum of NR12S is well matched to the emission spectrum of FITC, and its emission spectrum is polarity-sensitive, potentially allowing discrimination of interactions occurring in membrane domains with different degrees of liquid order, e.g., lipid rafts versus nonraft regions. The NR12S spectra obtained from excitation of FITC ligands incubated with NR12S-labeled cells showed an increase in maximum FRET signal without any significant spectral shift (Fig. 4B). Similar findings were observed with or without GLP-1R expression in the same cell system (Fig. 4B). This suggests that exendin-4-C16 may indeed interact with cell membranes to a greater extent than does exendin- 4 but does not provide evidence that this directs the ligand to interact with specific GLP-1R subpopulations situated in membrane nanodomains with different degrees of liquid order.

We also investigated the possibility of ligand-specific GLP-1R cAMP signal localization using the targeted cAMP FRET biosensor AKAP79-CUTie (Surdo et al., 2017). AKAP79 is a protein kinase A anchoring protein that is typically situated in membrane rafts by virtue of its palmitoylation (Delint-Ramirez et al., 2011). FRET imaging indicated similar kinetics for nanodomain-specific cAMP production with a saturating concentration of exendin-4 and exendin-4-C16 (Fig. 4C). Comparisons of ligand potencies for AKAP79-CUTie versus total cAMP measured by HTRF indicated that exendin-4-induced cAMP signaling is more constrained to AKAP79-marked nanodomains than for exendin-4-C16 (Fig. 4D; Supplemental Fig. 4C). On the other hand, pretreatment with methyl- $\beta$-cyclodextrin $(\mathrm{M} / \mathrm{CD})$ to sequester cholesterol and disrupt membrane nanodomain structure (Mahammad and Parmryd, 2015) disproportionately reduced cAMP production by exendin-4-C16 compared with exendin-4 (Fig. 4E). This might result from the lower $\mathrm{G}$ protein recruitment efficacy of exendin-4-C16, meaning that its ability to generate cAMP signals is more susceptible to uncoupling of $\mathrm{G}$ proteins from receptors as a result of disruption of membrane structure (Mystek et al., 2016). Similarly, exendin-4-C16-induced GLP-1R endocytosis, measured by TR-FRET, was more substantially affected by $\mathrm{M} \beta \mathrm{CD}$ treatment than exendin-4 (Fig. 4F).

To gain further insights into how exendin-4 and exendin-4C16 interact with GLP-1R at the plasma membrane, we used NR12S as a FRET acceptor for the N-terminally SNAPtagged GLP-1R labeled with Lumi-4-Tb, monitoring dynamic changes in TR-FRET signal with each ligand over time indicative of movement of the receptor ECD relative to the plasma membrane (Fig. 4G). A similar principle has been used previously to monitor epidermal growth factor receptor conformational shifts by FRET microscopy (Ziomkiewicz et al., 2013). Here, both ligands led to clear increases in TR-FRET signal across a wide concentration range. Efficacy was reduced with exendin-4-C16 (Fig. 4G), in keeping with lower efficacy measurements for intracellular responses including recruitment of mini- $\mathrm{G}_{\mathrm{s}}$ and $\beta$-arrestin-2 (see Fig. 1), although an increase in potency was observed. The implications of these responses are not clear, as they could reflect differences in ECD movement, known to be a feature of GLP-1R activation as determined from structural and computational studies (Zhang et al., 2019; Wu et al., 2020), but ligand-induced effects on membrane architecture cannot be ruled out.

Interaction of Exendin-4-C16 with Albumin and In Vivo Efficacy. The classic benefit of peptide acylation is to promote reversible binding to albumin in the circulation, thereby avoiding renal elimination. We previously showed that exendin-4-C16 and related analogs are $>97 \%$ bound to proteins in human and mouse plasma (Lucey et al., 2020). In the present study, we performed fluorescence correlation spectroscopy to measure the interaction of both TMR-conjugated peptides with BSA (Fig. 5, A-C). All traces could be fitted to a $1 \times 3 \mathrm{D}$ free diffusion model, indicating there is a single diffusing component. Diffusion coefficients for exendin4-TMR and exendin-4-TMR-C16, tested at a range of concentrations between 2.5 and $20 \mathrm{nM}$, were similar in the absence of BSA (Fig. 5B). However, diffusion of exendin-4-TMR-C16

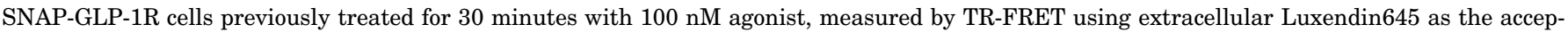

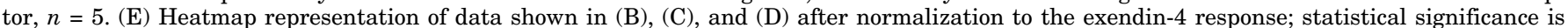

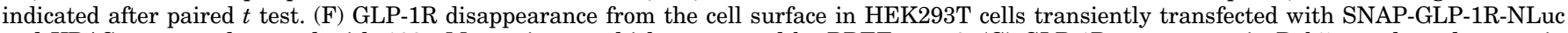

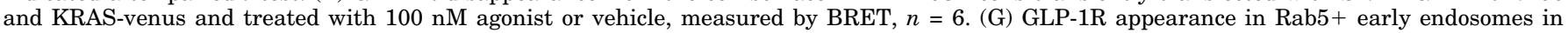

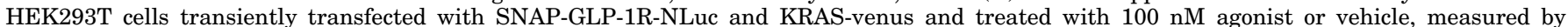

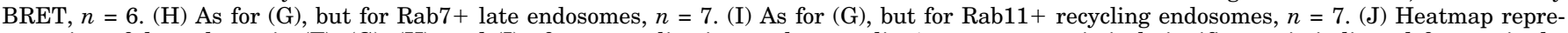

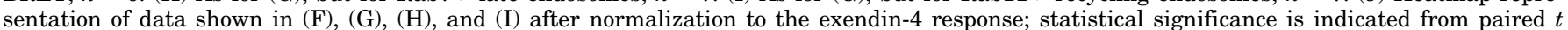

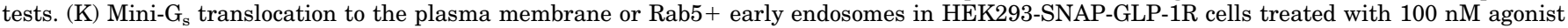

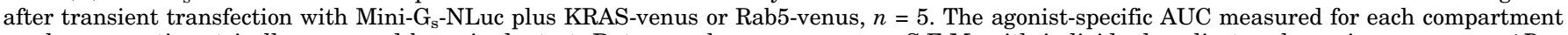

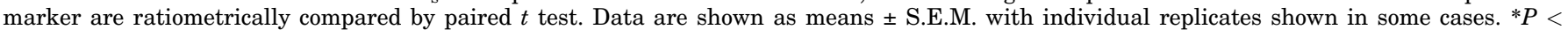
0.05 by statistical test indicated. 
A
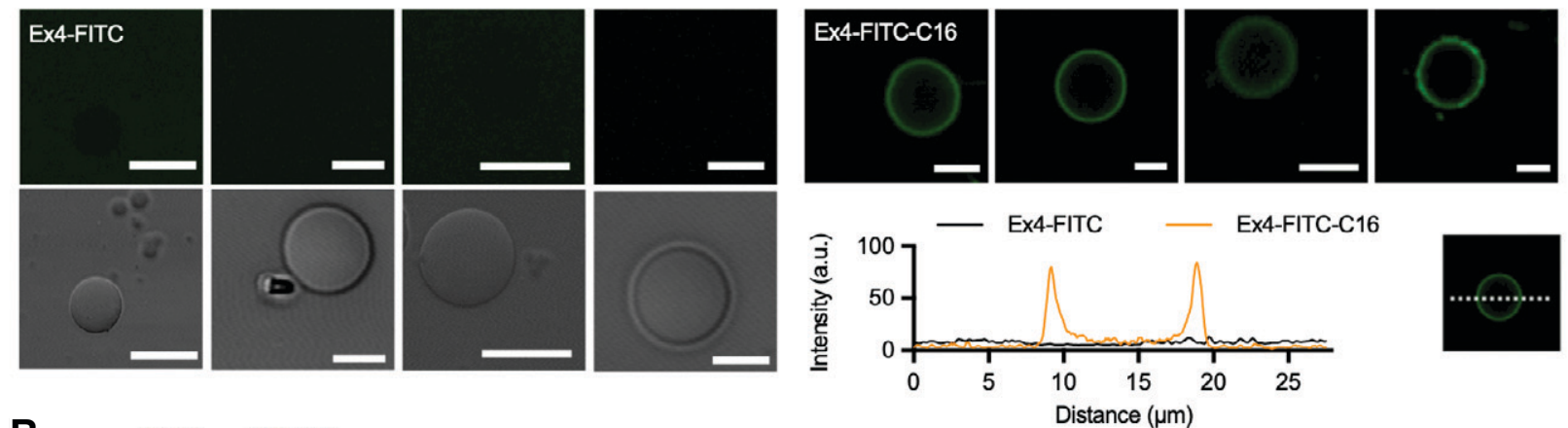

B
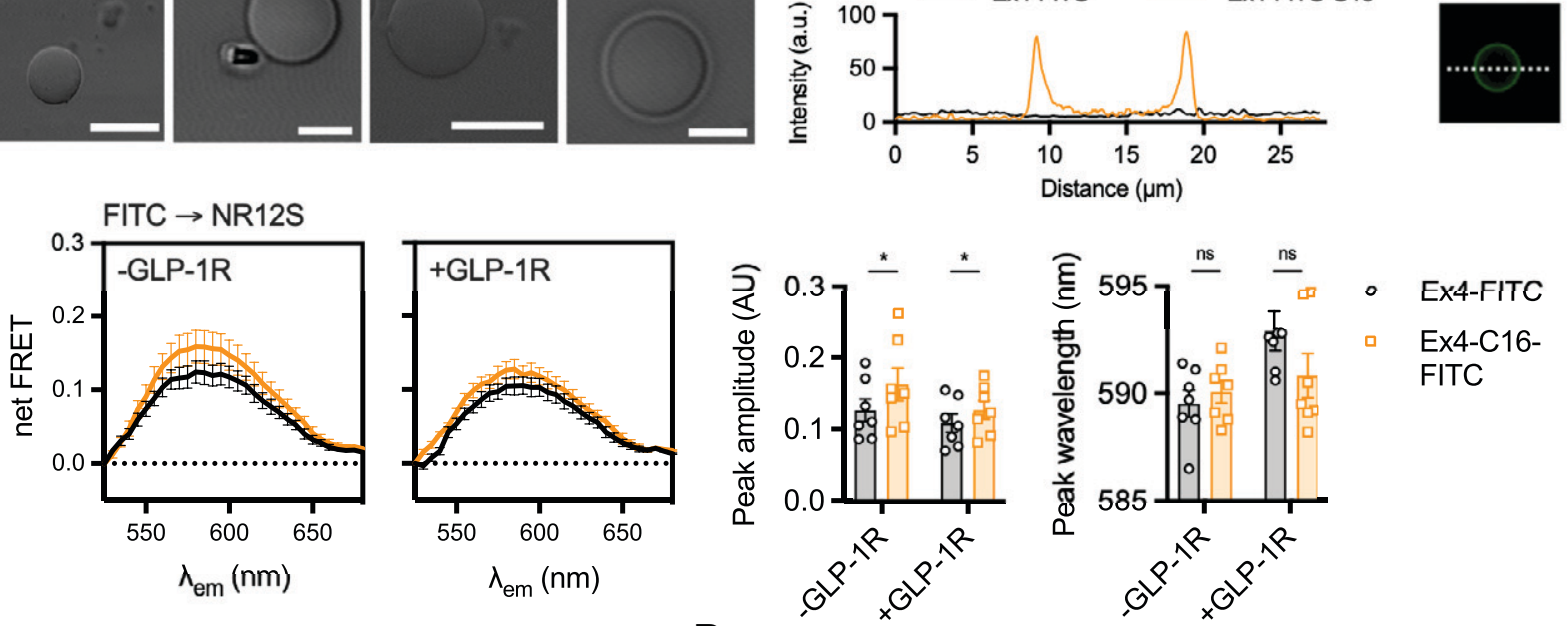

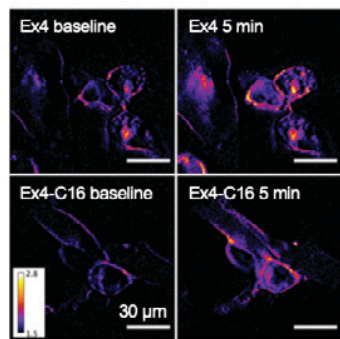

E
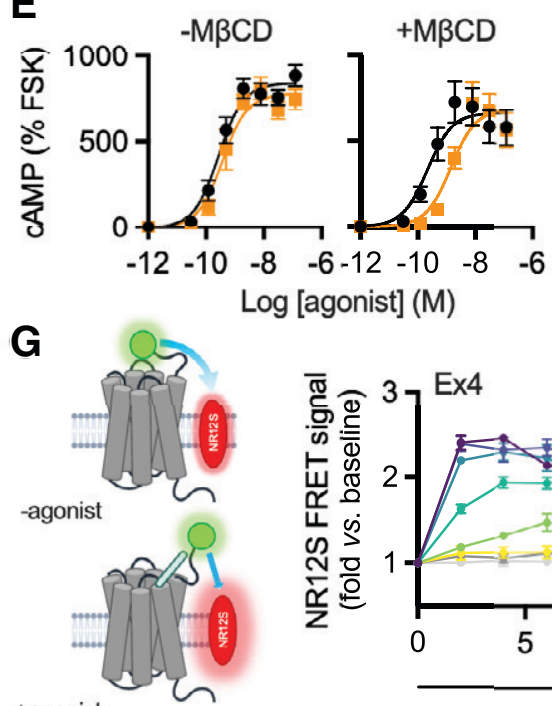

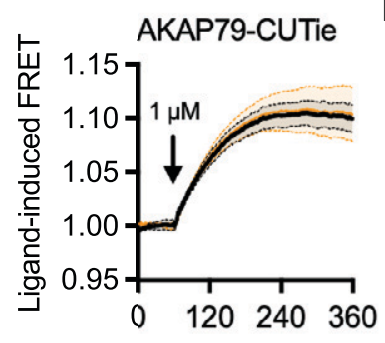

Time (s)
D

AKAP79-CUTie

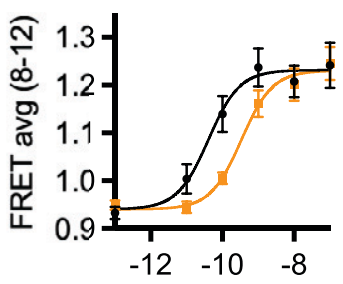

Log [agonist] (M)

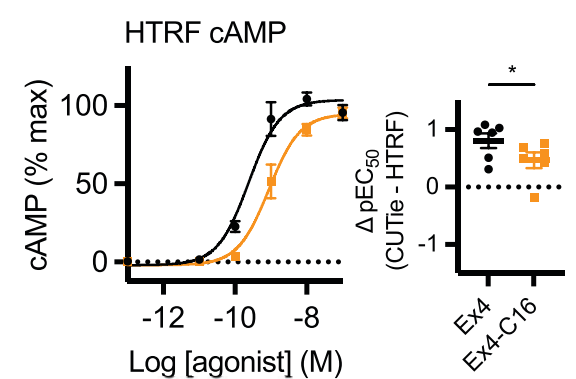

Ex4-FITC

Ex4-C16-

FITC
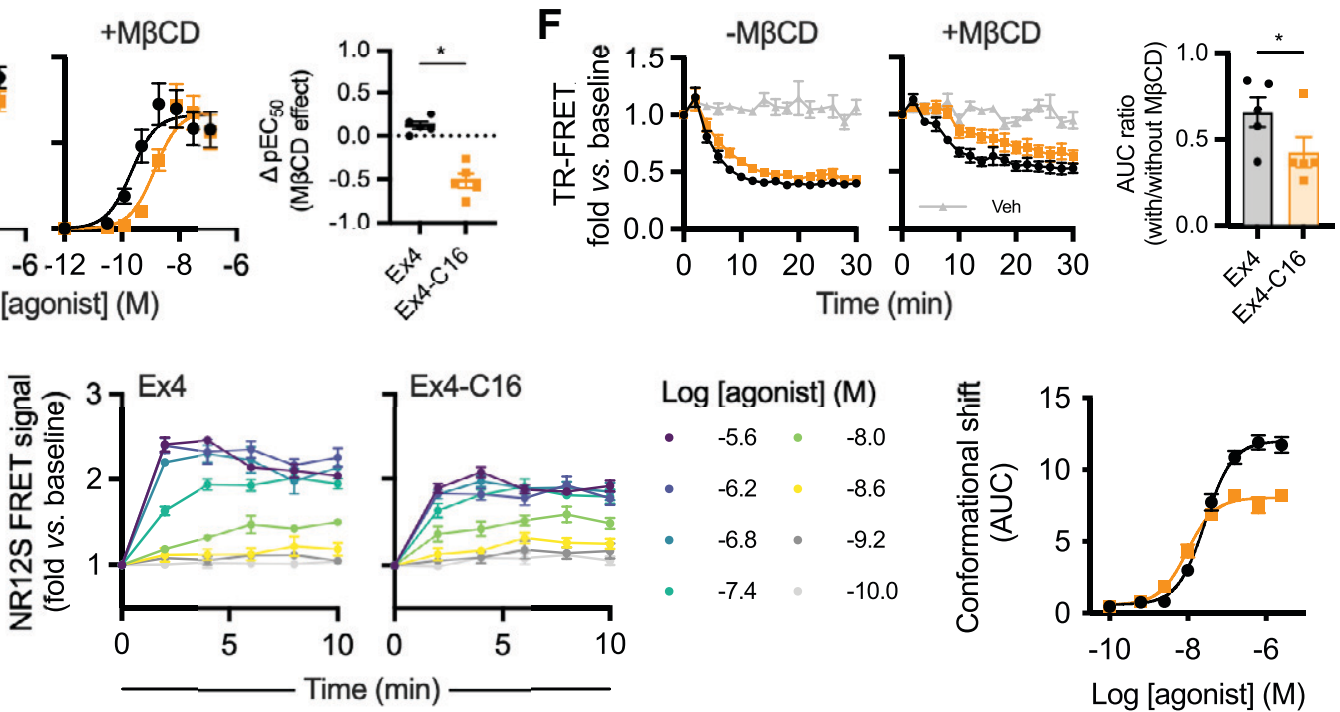

Fig. 4. Membrane interactions of C-terminally acylated exendin-4 conjugates. (A) Confocal microscopy and phase contrast images of GUVs incubated with exendin-4-FITC or exendin-4-FITC-C16. Scale bars, $5 \mu \mathrm{m}$. Membrane signal is represented on the line plot. (B) FRET spectrum from T-REx-SNAP-GLP-1R cells with or without tetracycline-induced GLP-1R expression, labeled with $50 \mathrm{nM}$ NR12S and incubated with $100 \mathrm{nM}$ exendin-4-FITC or exendin-4-FITC-C16, $n=7$. The background spectrum of each FITC ligand was subtracted, and the trace was normalized to the signal at $525 \mathrm{~nm}$. Peak emission wavelength and amplitude, derived from Gaussian fitting of each spectrum, are shown and compared between ligands by two-way randomized block ANOVA with Sidak's test. (C) FRET imaging of T-REx-SNAP-GLP-1R cells stimulated with $1 \mu$ M exendin-4 or exendin-4-C16, with representative ratiometric images pre- and poststimulation, and quantification from $n=3$ experiments. (D) Matched comparison of nanodomain-specific cAMP (AKAP79-CUTie) and total cAMP (HTRF) in T-REx-SNAP-GLP-1R cells, 12-minute stimulation, $n=6$, with 
A Ex4-TMR

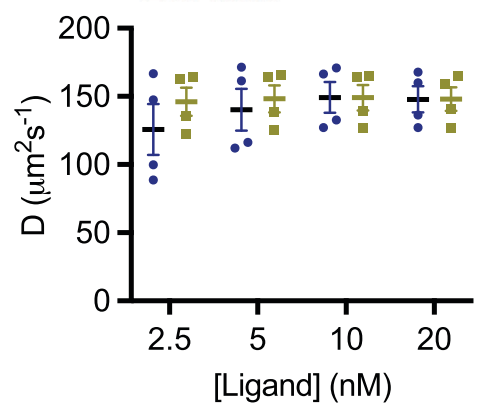

D

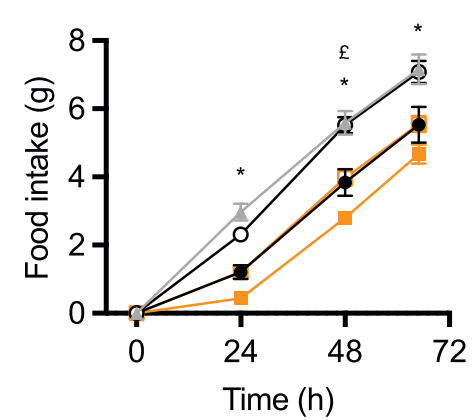

F

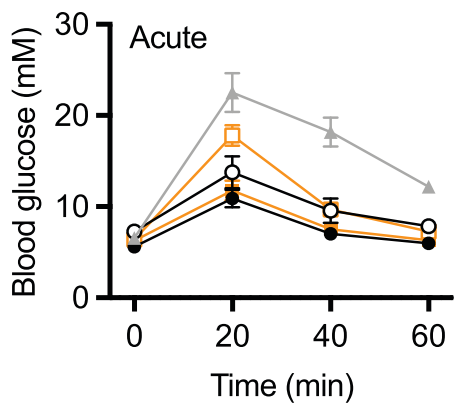

Ex4-TMR-C16

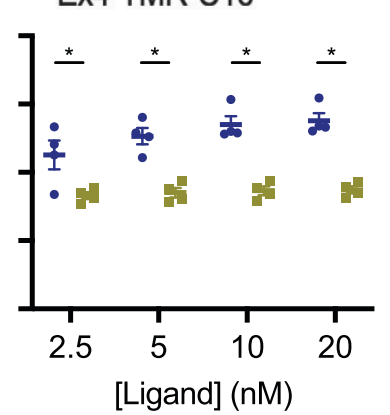

B

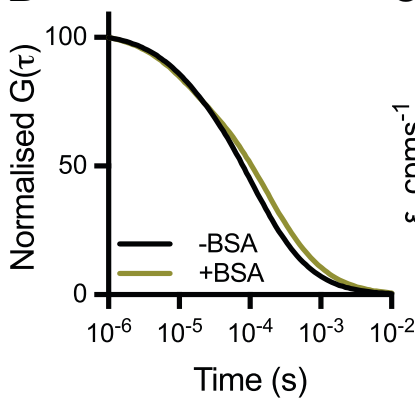

C

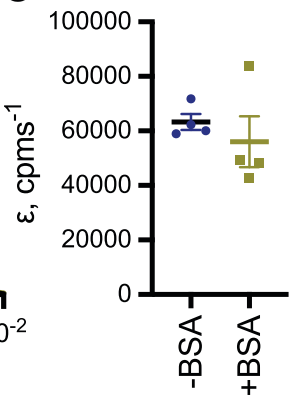

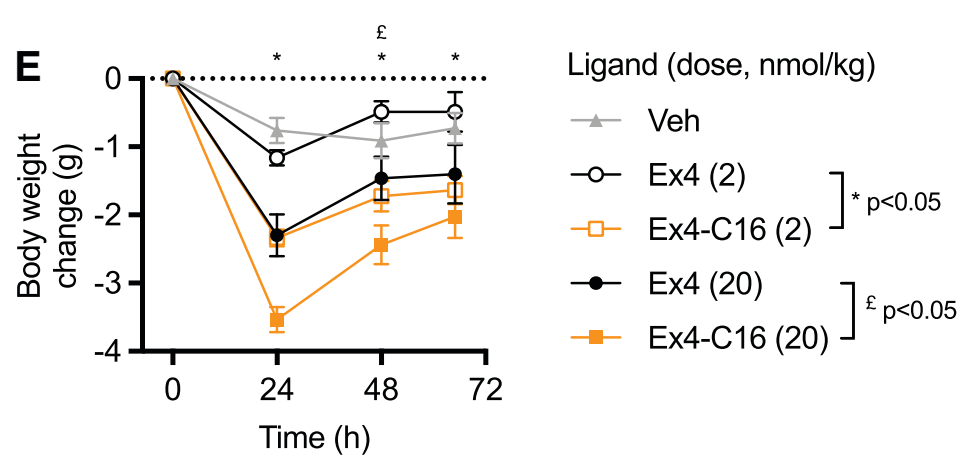
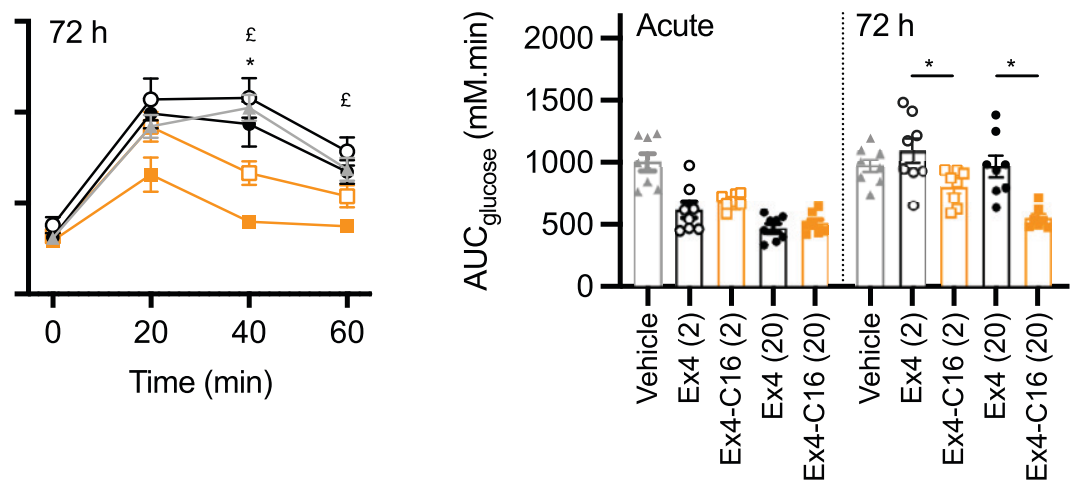

Fig. 5. Albumin binding by fluorescence correlation spectroscopy and in vivo effects. (A) Diffusion coefficients, $D\left(\mu m^{2} s^{-1}\right)$, of indicated concentration of indicated ligand with and without $0.1 \%$ BSA, with comparison by two-way randomized block ANOVA with Sidak's test. (B) Representative 1x3D free diffusion fit for Exendin4-TMR-C16 $(20 \mathrm{nM})$ autocorrelation curves with or without $0.1 \%$ BSA. The autocorrelation function, $\mathrm{G}(\tau)$, is normalized to facilitate comparison of decay curves. (C) Molecular brightness, $\varepsilon$ (counts per molecule per second), of EX4TMR-C16 $(20 \mathrm{nM})$ with and without $0.1 \%$ BSA. (D) Cumulative food intake in male HFD mice $(n=8 /$ group $)$ after a single intraperitoneal injection of the indicated agonist. Statistical comparisons between equimolar agonist doses by two-way repeated measures ANOVA with Tukey's test. (E) As for (B), but showing the effect on body weight. (F) IPGTT ( $2 \mathrm{~g} / \mathrm{kg}$ glucose) performed at the start and end of the 72-hour study. AUCs are compared by one-way ANOVA with Sidak's test for equimolar agonist doses. Data are shown as means \pm S.E.M. with individual replicates shown in some cases. ${ }^{*} P<0.05$ by statistical test indicated.

was markedly slowed in the presence of BSA, indicative of formation of peptide-albumin complexes. (Fig. 5, A and B). Exendin-4-TMR-C16 measurement reads were reanalyzed by $\mathrm{PCH}$ analysis with all reads fitting to a single-species $\mathrm{PCH}$ fit and the reported average molecular brightness unaffected by the addition of BSA (Fig. 5C). These results support our interpretation of a slowing of diffusion coefficient as a result of exendin-4-TMR-C16 interaction with BSA rather than an accumulation of peptide, which would have resulted in an increase in average molecular brightness.

Finally, the metabolic effects of exendin-4 and exendin-4C16 were compared in mice in which glucose intolerance was

$\Delta \mathrm{pEC}_{50}$ comparison by paired $t$ test. (E) cAMP responses in HEK293-SNAP-GLP-1R cells preincubated for 30 minutes with vehicle or 10 mM $\mathrm{M} \beta \mathrm{CD}$ prior to stimulation with each ligand for 30 minutes. Responses are expressed relative to the forskolin (FSK; $10 \mu \mathrm{M})$ response, $n=5$, with the effect of $\mathrm{M} \beta \mathrm{CD}$ on potency shown by subtracting $\mathrm{pEC}_{50}$ results and comparison by paired $t$ test. (F) GLP-1R internalization measured by DERET in HEK293-SNAP-GLP-1R cells preincubated for 30 minutes with vehicle or $10 \mathrm{mM} \mathrm{M} \beta \mathrm{CD}$ prior to stimulation with each ligand at 100 $\mathrm{nM}$ for 30 minutes, $n=5$. AUC relative to baseline was quantified, and $+\mathrm{M} \beta \mathrm{CD}$ results are expressed relative to $-\mathrm{M} \beta \mathrm{CD}$ results for each ligand, and compared by paired $t$ test. (G) Principle of NR12S conformational biosensor assay, with kinetic responses showing TR-FRET ratio normalized to baseline in response to indicated concentration of exendin-4 or exendin-4-C16, $n=5$, with concentration-response curve constructed from AUC with three-parameter fit. Data are shown as means \pm S.E.M. with individual replicates when possible. $* P<0.05$ by statistical test indicated. 
first induced by high-fat feeding for 2 months prior to the study. Exendin-4-C16 was previously shown to be detectable in plasma 72 hours after dosing (Lucey et al., 2020). Therefore, the effect of a single administration of each ligand, at two separate doses, was assessed. Both doses of exendin-4C16 led to greater suppression of food intake and weight loss throughout the dosing period (Fig. 5, D and E). Moreover, both doses of the acylated ligand exerted a larger antihyperglycemic effect in an intraperitoneal glucose tolerance test performed 72 hours after dosing (Fig. 5F).

\section{Discussion}

This study demonstrates how C-terminal acylation of exendin-4 affects several GLP-1R pharmacological properties that are relevant to its therapeutic effect. Although exendin-4-C16 showed minimal reduction in cAMP signaling potency, marked differences were observed for recruitment of key intracellular effectors and trafficking responses, which led to increased insulin secretion with prolonged incubations. We also evaluated the effect of peptide acylation on binding to membranes and albumin to more comprehensively describe the differences between these ligands. Overall, our study highlights the breadth of pharmacological parameters than can be affected by peptide acylation.

This work was prompted by our earlier observation that Cterminal acylation of biased GLP-1RAs based on exendin-4 results in reduced recruitment efficacy for $\beta$-arrestin and mini-G protein (Lucey et al., 2020). The present study confirms this observation, with a $60 \%$ reduction in maximum response recorded for $\beta$-arrestin- 2 recruitment by nanoBiT complementation with exendin-4-C16. Although mini-G recruitment was also reduced, the impact on $\beta$-arrestin- 2 recruitment was greater, with biased agonism confirmed by the operational model approach and on the basis of efficacy differences (Onaran et al., 2017). We note that this does not indicate that the ligands show opposing preference for G protein- versus $\beta$-arrestin-favoring GLP-1R conformations; the observed bias more likely results from the fact that $\beta$-arrestin responses appear to be more susceptible to reductions in GLP-1R efficacy than do G protein responses. Indeed, our data add to the growing body of evidence that $G$ protein-favoring biased GLP-1RAs typically show reduced efficacy for recruitment of both $\mathrm{G}$ proteins and $\beta$-arrestins (Fang et al., 2020b; Lucey et al., 2020; Pickford et al., 2020). However, using a novel Nb37-based BRET approach to monitor activation of endogenous $\mathrm{G}$ proteins close to GLP-1R, we demonstrate here that exendin-4-C16 shows increases in $\mathrm{G} \alpha_{\mathrm{s}}$ activation in spite of its lower mini- $G_{\mathrm{s}}$ recruitment efficacy. This could reflect an inherent difference in ability of exendin-4-C16 to activate $\mathrm{G} \alpha_{\mathrm{S}}$ in spite of reduced recruitment. However, we suspect the real reason is that the activation assay is more susceptible to normal regulatory processes, such as steric hindrance by $\beta$-arrestin recruitment, which fail to displace mini-G responses due to the highly stable nature of the GPCR-mini-G complexes (Carpenter and Tate, 2016). Although the dynamic range of this assay was low in our hands, making it challenging to apply to higher throughput screening efforts or to concentration responses, it could be adapted by the use of alternative fluorophores with greater signal separation from the nanoluciferase emission peak (Dale et al., 2019) or using complementation approaches (Inoue et al., 2019). Other approaches to monitor $\mathrm{G}$ protein activation have typically required overexpression of tagged $\mathrm{G}$ protein subunits (Masuho et al., 2015; Inoue et al., 2019; Olsen et al., 2020; Zhao et al., 2020), which may not totally replicate the physiologic setting, although corroboration using these approaches would be useful for validation.

In line with other studies showing that lower efficacy biased GLP-1RAs tend to induce slower GLP-1R endocytosis (Jones et al., 2018; Fremaux et al., 2019; Lucey et al., 2020; Willard et al., 2020), we observed that GLP-1R internalization was reduced with exendin-4-C16 compared with exendin-4. The automated microscopy approach we used to demonstrate this has certain advantages over lower throughput methods by allowing responses to a wide range of ligands or, in this case, ligand concentrations to be monitored in parallel across several fields of view, characterizing ligand effects in more detail and with greater statistical robustness. However, this method is unable to discriminate between GLP-1R clustering at the plasma membrane versus bona fide endocytosis events, although these are intrinsically linked, with the former occurring rapidly after ligand stimulation as a precursor to uptake into clathrin-coated vesicles (Buenaventura et al., 2019). The system could be adapted for use with alternative fluorescence approaches to monitor internalization, e.g., using pH-sensitive SNAP-labeling fluorophores (Martineau et al., 2017).

We also applied a series of complementary proximity-based techniques based on both BRET and FRET to monitor GLP$1 R$ redistribution between the plasma membrane and different endosomal compartments. Monitoring GLP-1R disappearance from the plasma membrane by DERET has been widely applied (Roed et al., 2014; Jones et al., 2018), and the use of the cleavable BG-Lumi4-Tb to monitor GLP-1R recycling in combination with a fluorescent antagonist ligand was recently described by our group (Pickford et al., 2020). We extended this approach here to detect GLP-1R translocation to late endosomes and lysosomes marked by the lysomotropic dye LysoTracker, facilitated by the spectral overlap of one of the $\mathrm{Tb}$ emission peaks with the excitation spectrum of LysoTracker DND99. In principle, a similar approach could be trialed with other fluorescent markers that accumulate in different subcellular compartments. Using a nanoluciferase tag at the GLP-1R C terminus, we were also able to obtain BRET measurements of GLP-1R redistribution to early, late, and recycling endosomes that corroborate the TR-FRET responses. This approach has been used recently to study the trafficking profiles of GLP-1R monoagonists and dual GLP-1R/ GIPR coagonists (Fletcher et al., 2018; Novikoff et al., 2021), albeit using Renilla luciferase rather than the nanoluciferase we used in our study. The rank order of ligand-induced changes was consistent for "matched" TR-FRET and BRET approaches, with exendin-4-C16 showing reduced GLP-1R internalization and lysosomal accumulation but faster recycling. In our hands. the TR-FRET approach resulted in clearer discrimination between ligand responses, which could reflect cell model differences, influence of the C-terminal NLuc tag for BRET, or assay/instrument sensitivity.

Importantly, we also observed differences in mini- $\mathrm{G}_{\mathrm{s}}$ recruitment to plasma membrane versus early endosomes using the nanoBRET approach, providing some insight into compartmentalization of GLP1-R signaling. Here, plasma membrane mini- $\mathrm{G}_{\mathrm{s}}$ recruitment was somewhat reduced for exendin-4-C16 compared with exendin-4, which is compatible 
with results from our nanoBiT complementation assay demonstrating reduced global mini- $\mathrm{G}_{\mathrm{s}}$ recruitment efficacy for this ligand. However, recruitment of mini- $\mathrm{G}_{\mathrm{s}}$ to Rab5-positive early endosomes was reduced to an even greater extent, which can be at least partly explained by the reduced rate of internalization with this ligand, although differences in ability to maintain GLP-1R activation once internalized could also contribute. Indeed, the GLP-1R-Rab5 BRET signal was only marginally reduced with exendin-4-C16 versus exendin4, whereas the mini- $\mathrm{G}_{\mathrm{s}}$-Rab5 BRET response showed larger differences between ligands. However, differences in luciferase/fluorophore configuration between assays, as well as unknown effects of mini- $\mathrm{G}_{\mathrm{s}}$ overexpression on GLP-1R pharmacology, mean that further work will be needed to fully explain this observation. GLP-1R has been reported to generate signals from the endosomal compartment (Kuna et al., 2013; Roed et al., 2015; Girada et al., 2017; Fletcher et al., 2018), in line with many other GPCRs (Vilardaga et al., 2014), and this phenomenon is frequently claimed to be a mechanism for sustained cAMP signaling. However, although our results corroborate the existence of GLP-1R-associated endosomal signaling, they also suggest that sustained GLP-1R signaling, as indicated by cumulative insulin secretion over pharmacologically relevant timescales, is actually greater with the ligand (exendin-4-C16) with a reduced tendency to recruit mini- $\mathrm{G}_{\mathrm{s}}$ to Rab5-positive endosomes. This raises questions about the relative therapeutic importance of maintaining an adequate pool of surface GLP-1Rs during prolonged stimulations versus aiming for maximal endosomal receptor activation.

The structural basis for the modified GLP-1R activation profile with exendin-4-C16 is not clear. The $\mathrm{C}$ terminus of exendin-4, although not required for GLP-1R activation (Lee et al., 2018), plays an important role in GLP-1R binding (Doyle et al., 2003). Installation of a large acyl chain at the peptide $\mathrm{C}$ terminus could potentially interfere with ligand binding. We observed a modest reduction in GLP-1R binding affinity, in keeping with this possibility. However, receptor activation efficacy was also reduced. Interestingly, the $\mathrm{C}$ terminus of exendin-4 may be required to facilitate formation of high-order GLP-1R oligomers through interaction with neighboring GLP-1R protomers in trans (Koole et al., 2017). GLP-1R oligomerization is reported to be required for full signaling responses (Harikumar et al., 2012). A further possibility is that the acyl chain could interact with the plasma membrane in a specific manner that interferes with GLP-1R activation. We found here that a fluorescently labeled exendin-4-C16 does indeed form interactions with model membranes, whereas the equivalently labeled nonacylated exendin- 4 does not. Corresponding measurements from living cells also suggested greater membrane interactions with the acylated ligand but did not support the possibility of localized activation of GLP-1R subpopulations situated in particular plasma membrane nanodomains.

As differential ability of ligands to stabilize active GLP-1R conformations is a further potential explanation for the ligand signaling efficacy differences, we devised a strategy to monitor movements between the receptor ECD and the plasma membrane, finding efficacy reductions with exendin4-C16 that matched the reduced intracellular signaling responses also observed with this ligand. This approach may be more widely useful as a conformational sensor for other GPCRs, although it lacks the ability to detect changes at the receptor intracellular face that is needed to provide insights into conformational changes required for $G$ protein interactions.

There are a number of limitations with our study. Firstly, we investigated the effects of a single type of $\mathrm{C} 16$ diacid acyl chain, and our results are not necessarily extrapolable to GLP-1R agonists with different acyl chain lengths or acyl monoacids. Secondly, the majority of studies were performed in heterologous cell lines with overexpression of tagged GLP1R constructs, which could influence the pharmacology. Although numerous studies have demonstrated that biased agonism and trafficking assessments in heterologous systems reliably predict insulin secretory and other key physiologic effects of biased GLP-1RAs in vivo, the field would benefit from further efforts to study behaviors of endogenous GLP-1R in $\beta$ cells and cell types with native GLP-1R expression.

In summary, beyond the expected effects on binding to albumin, C-terminal acylation of exendin-4 led to changes in multiple pharmacological parameters relevant to downstream GLP$1 \mathrm{R}$ responses. These observations are more broadly relevant to drug discovery at peptide GPCRs for which ligand acylation is a valid approach to improve pharmacokinetics.

\section{Authorship Contributions}

Participated in research design: Lucey, Wang, Goulding, Minnion, Elani, Briddon, Bloom, Tomas, Jones.

Conducted experiments: Lucey, Ashik, Marzook, Wang, Goulding, Elani, Jones.

Contributed new reagents or analytic tools: Marzook, Oishi, Broichhagen, Hodson, Jockers.

Performed data analysis: Lucey, Ashik, Goulding, Elani, Jones.

Wrote or contributed to the writing of the manuscript: Lucey, Ashik, Marzook, Wang, Goulding, Oishi, Broichhagen, Hodson, Minnion, Elani, Jockers, Briddon, Bloom, Tomas, Jones.

\section{References}

Andersen A, Lund A, Knop FK, and Vilsbøll T (2018) Glucagon-like peptide 1 in health and disease. Nat Rev Endocrinol 14:390-403.

Andersen NH, Brodsky Y, Neidigh JW, and Prickett KS (2002) Medium-dependence of the secondary structure of exendin-4 and glucagon-like-peptide-1. Bioorg Med Chem 10:79-85.

Ast J, Arvaniti A, Fine NHF, Nasteska D, Ashford FB, Stamataki Z, Koszegi Z, Bacon A, Jones BJ, Lucey MA, et al. (2020) Super-resolution microscopy compatible fluorescent probes reveal endogenous glucagon-like peptide-1 receptor distribution and dynamics. Nat Commun 11:467.

Buenaventura T, Bitsi S, Laughlin WE, Burgoyne T, Lyu Z, Oqua AI, Norman H, McGlone ER, Klymchenko AS, Corrêa Jr IR, et al. (2019) Agonist-induced membrane nanodomain clustering drives GLP-1 receptor responses in pancreatic beta cells. PLoS Biol 17:e3000097.

Carpenter B and Tate CG (2016) Engineering a minimal G protein to facilitate crystallisation of G protein-coupled receptors in their active conformation. Protein Eng Des Sel 29:583-594.

Clardy SM, Keliher EJ, Mohan JF, Sebas M, Benoist C, Mathis D, and Weissleder R (2014) Fluorescent exendin-4 derivatives for pancreatic $\beta$-cell analysis. Bioconjug Chem 25:171-177.

Corriden R, Kilpatrick LE, Kellam B, Briddon SJ, and Hill SJ (2014) Kinetic analysis of antagonist-occupied adenosine-A3 receptors within membrane microdomains of individual cells provides evidence of receptor dimerization and allosterism. FASEB $J$ 28:4211-4222.

Curtis MJ, Alexander S, Cirino G, Docherty JR, George CH, Giembycz MA, Hoyer D, Insel PA, Izzo AA, Ji Y, et al. (2018) Experimental design and analysis and their reporting II: updated and simplified guidance for authors and peer reviewers. $B r J$ Pharmacol 175:987-993.

Dale NC, Johnstone EKM, White CW, and Pfleger KDG (2019) NanoBRET: the bright future of proximity-based assays. Front Bioeng Biotechnol 7:56.

Delint-Ramirez I, Willoughby D, Hammond GRV, Ayling LJ, Cooper DM, and Cooper DMF (2011) Palmitoylation targets AKAP79 protein to lipid rafts and promotes its regulation of calcium-sensitive adenylyl cyclase type 8. J Biol Chem 286:32962-32975.

Dixon AS, Schwinn MK, Hall MP, Zimmerman K, Otto P, Lubben TH, Butler BL, Binkowski BF, Machleidt T, Kirkland TA, et al. (2016) NanoLuc complementation 
reporter optimized for accurate measurement of protein interactions in cells. ACS Chem Biol 11:400-408.

Doyle ME, Theodorakis MJ, Holloway HW, Bernier M, Greig NH, and Egan JM (2003) The importance of the nine-amino acid C-terminal sequence of exendin- 4 for binding to the GLP-1 receptor and for biological activity. Regul Pept 114:153-158.

Fang Z, Chen S, Manchanda Y, Bitsi S, Pickford P, David A, Shchepinova MM, Corrêa Jr IR, Hodson DJ, Broichhagen J, et al. (2020a) Ligand-specific factors influencing GLP-1 receptor post-endocytic trafficking and degradation in pancreatic beta cells. Int J Mol Sci 21:8404.

Fang Z, Chen S, Pickford P, Broichhagen J, Hodson DJ, Corrêa Jr IR, Kumar S, Görlitz F, Dunsby C, French PMW, et al. (2020b) The influence of peptide context on signaling and trafficking of glucagon-like peptide-1 receptor biased agonists ACS Pharmacol Transl Sci 3:345-360.

Fletcher MM, Halls ML, Zhao P, Clydesdale L, Christopoulos A, Sexton PM, and Wootten D (2018) Glucagon-like peptide-1 receptor internalisation controls spatiotemporal signalling mediated by biased agonists. Biochem Pharmacol 156:406-419.

Fox CB, Wayment JR, Myers GA, Endicott SK, and Harris JM (2009) Single-molecule fluorescence imaging of peptide binding to supported lipid bilayers. Anal Chem 81:5130-5138.

Fremaux J, Venin C, Mauran L, Zimmer R, Koensgen F, Rognan D, Bitsi S, Lucey MA, Jones B, Tomas A, et al. (2019) Ureidopeptide GLP-1 analogues with prolonged activity in vivo via signal bias and altered receptor trafficking. Chem Sci (Camb) 10:9872-9879.

Girada SB, Kuna RS, Bele S, Zhu Z, Chakravarthi NR, DiMarchi RD, and Mitra P (2017) $\mathrm{G} \alpha$ s regulates glucagon-like peptide 1 receptor-mediated cyclic AMP generation at Rab5 endosomal compartment. Mol Metab 6:1173-1185.

Graaf Cd, Donnelly D, Wootten D, Lau J, Sexton PM, Miller LJ, Ahn J-M, Liao J, Fletcher MM, Yang D, et al. (2016) Glucagon-like peptide-1 and its class B g protein-coupled receptors: a long march to therapeutic successes. Pharmacol Rev 68:954-1013.

Guariguata L, Whiting DR, Hambleton I, Beagley J, Linnenkamp U, and Shaw JE (2014) Global estimates of diabetes prevalence for 2013 and projections for 2035 Diabetes Res Clin Pract 103:137-149.

Harikumar KG, Wootten D, Pinon DI, Koole C, Ball AM, Furness SGB, Graham B, Dong M, Christopoulos A, Miller LJ, et al. (2012) Glucagon-like peptide-1 receptor dimerization differentially regulates agonist signaling but does not affect small molecule allostery. Proc Natl Acad Sci USA 109:18607-18612.

Hohmeier HE, Mulder H, Chen G, Henkel-Rieger R, Prentki M, and Newgard CB (2000) Isolation of INS-1-derived cell lines with robust ATP-sensitive K+ channeldependent and -independent glucose-stimulated insulin secretion. Diabetes 49:424-430.

Huang B, Perroud TD, and Zare RN (2004) Photon counting histogram: one-photon excitation. ChemPhysChem 5:1523-1531.

Inoue A, Raimondi F, Kadji FMN, Singh G, Kishi T, Uwamizu A, Ono Y, Shinjo Y, Ishida S, Arang N, et al. (2019) Illuminating G-protein-coupling selectivity of GPCRs. Cell 177:1933-1947.e25.

Irannejad R, Tomshine JC, Tomshine JR, Chevalier M, Mahoney JP, Steyaert J, Rasmussen SGF, Sunahara RK, El-Samad H, Huang B, et al. (2013) Conformational biosensors reveal GPCR signalling from endosomes. Nature 495:534-538.

Jaccard N, Griffin LD, Keser A, Macown RJ, Super A, Veraitch FS, and Szita N (2014) Automated method for the rapid and precise estimation of adherent cell culture characteristics from phase contrast microscopy images. Biotechnol Bioeng 111:504-517.

Jones B, Buenaventura T, Kanda N, Chabosseau P, Owen BM, Scott R, Goldin R, Angkathunyakul N, Corrêa Jr IR, Bosco D, et al. (2018) Targeting GLP-1 receptor trafficking to improve agonist efficacy. Nat Commun 9:1602.

Kenakin T, Watson C, Muniz-Medina V, Christopoulos A, and Novick S (2012) A simple method for quantifying functional selectivity and agonist bias. ACS Chem Neurosci 3:193-203.

Knudsen LB and Lau J (2019) The discovery and development of liraglutide and semaglutide. Front Endocrinol (Lausanne) 10:155

Kolterman OG, Buse JB, Fineman MS, Gaines E, Heintz S, Bicsak TA, Taylor K, Kim D, Aisporna M, Wang Y, et al. (2003) Synthetic exendin-4 (exenatide) significantly reduces postprandial and fasting plasma glucose in subjects with type 2 diabetes. $J$ Clin Endocrinol Metab 88:3082-3089.

Koole C, Reynolds CA, Mobarec JC, Hick C, Sexton PM, and Sakmar TP (2017) Genetically encoded photocross-linkers determine the biological binding site of exendin-4 peptide in the N-terminal domain of the intact human glucagon-like peptide-1 receptor (GLP-1R). J Biol Chem 292:7131-7144.

Kucherak OA, Oncul S, Darwich Z, Yushchenko DA, Arntz Y, Didier P, Mély Y, and Klymchenko AS (2010) Switchable nile red-based probe for cholesterol and lipid order at the outer leaflet of biomembranes. J Am Chem Soc 132:4907-4916.

Kuna RS, Girada SB, Asalla S, Vallentyne J, Maddika S, Patterson JT, Smiley DL, DiMarchi RD, and Mitra P (2013) Glucagon-like peptide-1 receptor-mediated endosomal cAMP generation promotes glucose-stimulated insulin secretion in pancreatic B-cells. Am J Physiol Endocrinol Metab 305:E161-E170.

Lau J, Bloch P, Schäffer L, Pettersson I, Spetzler J, Kofoed J, Madsen K, Knudsen LB, McGuire J, Steensgaard DB, et al. (2015) Discovery of the once-weekly glucagon-like peptide-1 (GLP-1) analogue semaglutide. J Med Chem 58:7370-7380.

Lee JG, Ryu JH, Kim S-M, Park M-Y, Kim S-H, Shin YG, Sohn J-W, Kim HH, Park Z-Y, Seong JY, et al. (2018) Replacement of the C-terminal Trp-cage of exendin-4 with a fatty acid improves therapeutic utility. Biochem Pharmacol 151:59-68.

Levoye A, Zwier JM, Jaracz-Ros A, Klipfel L, Cottet M, Maurel D, Bdioui S, Balabanian K, Prézeau L, Trinquet E, et al. (2015) A broad G protein-coupled receptor internalization assay that combines SNAP-Tag labeling, diffusion-enhanced resonance energy transfer, and a highly emissive terbium cryptate. Front Endocrinol (Lausanne) 6:167.

Lucey M, Pickford P, Bitsi S, Minnion J, Ungewiss J, Schoeneberg K, Rutter GA Bloom SR, Tomas A, and Jones B (2020) Disconnect between signalling potency and in vivo efficacy of pharmacokinetically optimised biased glucagon-like peptide-1 receptor agonists. Mol Metab 37:100991.

Mahammad S and Parmryd I (2015) Cholesterol depletion using methyl- $\beta$-cyclodextrin. Methods Mol Biol 1232:91-102.

Malm-Erjefält M, Bjørnsdottir I, Vanggaard J, Helleberg H, Larsen U, Oosterhuis B, van Lier JJ, Zdravkovic M, and Olsen AK (2010) Metabolism and excretion of the once-daily human glucagon-like peptide-1 analog liraglutide in healthy male subjects and its in vitro degradation by dipeptidyl peptidase IV and neutral endopeptidase. Drug Metab Dispos 38:1944-1953.

Manchanda Y, Bitsi S, Kang Y, Jones B, and Tomas A (2021) Spatiotemporal control of GLP-1 receptor activity. Curr Opin Endocr Metab Res 16:19-27.

Martineau M, Somasundaram A, Grimm JB, Gruber TD, Choquet D, Taraska JW, Lavis LD, and Perrais D (2017) Semisynthetic fluorescent $\mathrm{pH}$ sensors for imaging exocytosis and endocytosis. Nat Commun 8:1412.

Masuho I, Ostrovskaya O, Kramer GM, Jones CD, Xie K, and Martemyanov KA (2015) Distinct profiles of functional discrimination among $\mathrm{G}$ proteins determine the actions of G protein-coupled receptors. Sci Signal 8:ra123.

Mystek P, Dutka P, Tworzydło M, Dziedzicka-Wasylewska M, and Polit A (2016) The role of cholesterol and sphingolipids in the dopamine $D_{1}$ receptor and $G$ protein distribution in the plasma membrane. Biochim Biophys Acta 1861:1775-1786.

Neidigh JW, Fesinmeyer RM, Prickett KS, and Andersen NH (2001) Exendin-4 and glucagon-like-peptide-1: NMR structural comparisons in the solution and micelleassociated states. Biochemistry 40:13188-13200.

Novikoff A, O’Brien SL, Bernecker M, Grandl G, Kleinert M, Knerr PJ, Stemmer K, Klingenspor M, Zeigerer A, DiMarchi R, et al. (2021) Spatiotemporal GLP-1 and GIP receptor signaling and trafficking/recycling dynamics induced by selected receptor mono- and dual-agonists. Mol Metab 49:101181.

Nuber S, Zabel U, Lorenz K, Nuber A, Milligan G, Tobin AB, Lohse MJ, and Hoffmann $C$ (2016) $\beta$-Arrestin biosensors reveal a rapid, receptor-dependent activation/ deactivation cycle. Nature 531:661-664.

Oishi A, Dam J, and Jockers R (2019) $\beta$-Arrestin-2 BRET biosensors detect different $\beta$-arrestin-2 conformations in interaction with GPCRs. ACS Sens 5:57-64.

Olsen RHJ, DiBerto JF, English JG, Glaudin AM, Krumm BE, Slocum ST, Che T, Gavin AC, McCorvy JD, Roth BL, et al. (2020) TRUPATH, an open-source biosensor platform for interrogating the GPCR transducerome. Nat Chem Biol 16:841-849.

Onaran HO, Ambrosio C, Uğur Ö, Madaras Koncz E, Grò MC, Vezzi V, Rajagopal $\mathrm{S}$, and Costa T (2017) Systematic errors in detecting biased agonism: Analysis of current methods and development of a new model-free approach. Sci Rep 7:44247.

Peng T, Thorn K, Schroeder T, Wang L, Theis FJ, Marr C, and Navab N (2017) A BaSiC tool for background and shading correction of optical microscopy images. Nat Commun 8:14836-14837.

Pickford P, Lucey M, Fang Z, Bitsi S, de la Serna JB, Broichhagen J, Hodson DJ, Minnion J, Rutter GA, Bloom SR, et al. (2020) Signalling, trafficking and glucoregulatory properties of glucagon-like peptide-1 receptor agonists exendin-4 and lixisenatide. Br J Pharmacol 177:3905-3923.

Roed SN, Nøhr AC, Wismann P, Iversen H, Bräuner-Osborne H, Knudsen SM, and Waldhoer M (2015) Functional consequences of glucagon-like peptide-1 receptor cross-talk and trafficking. J Biol Chem 290:1233-1243.

Roed SN, Wismann P, Underwood CR, Kulahin N, Iversen H, Cappelen KA, Schäffer L, Lehtonen J, Hecksher-Soerensen J, Secher A, et al. (2014) Real-time trafficking and signaling of the glucagon-like peptide-1 receptor. Mol Cell Endocrinol 382:938-949.

Sage D, Donati L, Soulez F, Fortun D, Schmit G, Seitz A, Guiet R, Vonesch C, and Unser M (2017) DeconvolutionLab2: an open-source software for deconvolution microscopy. Methods 115:28-41.

Surdo NC, Berrera M, Koschinski A, Brescia M, Machado MR, Carr C, Wright P, Gorelik J, Morotti S, Grandi E, et al. (2017) FRET biosensor uncovers cAMP nanodomains at $\beta$-adrenergic targets that dictate precise tuning of cardiac contractility. Nat Commun 8:15031.

Thornton K and Gorenstein DG (1994) Structure of glucagon-like peptide (7-36) amide in a dodecylphosphocholine micelle as determined by 2D NMR. Biochemistry 33:3532-3539.

Tiulpakov A, White CW, Abhayawardana RS, See HB, Chan AS, Seeber RM, Heng JI, Dedov I, Pavlos NJ, and Pfleger KDG (2016) Mutations of vasopressin receptor 2 including novel L312S have differential effects on trafficking. Mol Endocrinol 30:889-904.

Tomas A, Jones B, and Leech C (2019) New insights into beta-cell GLP-1 receptor and cAMP signaling. J Mol Biol 432:1347-1366.

Trier S, Linderoth L, Bjerregaard S, Andresen TL, and Rahbek UL (2014) Acylation of Glucagon-like peptide-2: interaction with lipid membranes and in vitro intestinal permeability. PLoS One 9:e109939.

van der Westhuizen ET, Breton B, Christopoulos A, and Bouvier M (2014) Quantification of ligand bias for clinically relevant $\beta 2$-adrenergic receptor ligands: implications for drug taxonomy. Mol Pharmacol 85:492-509.

Vilardaga J-P, Jean-Alphonse FG, and Gardella TJ (2014) Endosomal generation of cAMP in GPCR signaling. Nat Chem Biol 10:700-706.

Villar VAM, Cuevas S, Zheng X, and Jose PA (2016) Localization and signaling of GPCRs in lipid rafts. Methods Cell Biol 132:3-23.

Wan Q, Okashah N, Inoue A, Nehmé R, Carpenter B, Tate CG, and Lambert NA (2018) Mini G protein probes for active G protein-coupled receptors (GPCRs) in live cells. J Biol Chem 293:7466-7473.

Widmann C, Dolci W, and Thorens B (1995) Agonist-induced internalization and recycling of the glucagon-like peptide-1 receptor in transfected fibroblasts and in insulinomas. Biochem $J$ 310:203-214.

Willard FS, Douros JD, Gabe MB, Showalter AD, Wainscott DB, Suter TM, Capozzi ME, van der Velden WJ, Stutsman C, Cardona GR, et al. (2020) 
Tirzepatide is an imbalanced and biased dual GIP and GLP-1 receptor agonist. JCI Insight 5:1202.

Wu F, Yang L, Hang K, Laursen M, Wu L, Han GW, Ren Q, Roed NK, Lin G, Hanson MA, et al. (2020) Full-length human GLP-1 receptor structure without orthosteric ligands. Nat Commun 11:1272.

Zhang H, Sturchler E, Zhu J, Nieto A, Cistrone PA, Xie J, He L, Yea K, Jones T, Turn $\mathrm{R}$, et al. (2015) Autocrine selection of a GLP-1R G-protein biased agonist with potent antidiabetic effects. Nat Commun 6:8918.

Zhang J, Bai Q, Pérez-Sánchez H, Shang S, An X, and Yao X (2019) Investigation of ECD conformational transition mechanism of GLP-1R by molecular dynamics simulations and Markov state model. Phys Chem Chem Phys 21:8470-8481.

Zhao P, Liang Y-L, Belousoff MJ, Deganutti G, Fletcher MM, Willard FS, Bell MG, Christe ME, Sloop KW, Inoue A, et al. (2020) Activation of the GLP-1 receptor by a non-peptidic agonist. Nature 577:432-436.
Ziomkiewicz I, Loman A, Klement R, Fritsch C, Klymchenko AS, Bunt G, Jovin TM, and Arndt-Jovin DJ (2013) Dynamic conformational transitions of the EGF receptor in living mammalian cells determined by FRET and fluorescence lifetime imaging microscopy. Cytometry A 83:794-805.

Address correspondence to: Ben Jones, Section of Endocrinology and Investigative Medicine, Department of Metabolism, Digestion and Reproduction, Imperial College London, London, W12 0NN, United Kingdom. E-mail: ben.jones@ imperial.ac.uk; or Alejandra Tomas, Section of Cell Biology and Functional Genomics, Department of Metabolism, Digestion and Reproduction, Imperial College London, London, W12 0NN, United Kingdom. E-mail: a.tomas-catala@ imperial.ac.uk 


\section{Supplementary Information}

\section{\#MOLPHARM-AR-2021-000270R1}

Acylation of the incretin peptide exendin-4 directly impacts GLP-1 receptor signalling and trafficking

Maria Lucey ${ }^{1}$, Tanyel Ashik ${ }^{1}$, Amaara Marzook ${ }^{1}$, Yifan Wang ${ }^{2}$, Joëlle Goulding ${ }^{3,4}$, Atsuro Oishi $^{5,6}$, Johannes Broichhagen ${ }^{7}$, David J Hodson ${ }^{4,8,9}$, James Minnion ${ }^{1}$, Yuval Elani ${ }^{10}$, Ralf Jockers $^{5}$, Stephen J Briddon ${ }^{3,4}$, Stephen R Bloom ${ }^{1}$, Alejandra Tomas ${ }^{2, ~ \#, ~ B e n ~ J o n e s ~}{ }^{1, \# . ~}$

${ }^{*}$ Co-senior authors

${ }^{1}$ Section of Endocrinology and Investigative Medicine, Department of Metabolism, Digestion and Reproduction, Imperial College London, London, W12 ONN, UK.

${ }^{2}$ Section of Cell Biology and Functional Genomics, Department of Metabolism, Digestion and Reproduction, Imperial College London, London, W12 0NN, UK.

3 Division of Physiology, Pharmacology and Neuroscience, School of Life Sciences, University of Nottingham, Nottingham, NG7 2UH, UK.

${ }^{4}$ Centre of Membrane Proteins and Receptors (COMPARE), University of Birmingham and University of Nottingham, Midlands, UK.

${ }^{5}$ Université de Paris, Institut Cochin, INSERM, CNRS, F-75014 Paris, France.

${ }^{6}$ Department of Anatomy, Kyorin University Faculty of Medicine, Tokyo, Japan.

7 Leibniz-Forschungsinstitut für Molekulare Pharmakologie, Robert-Rössle-Str. 10, 13125 Berlin, Germany.

8 Institute of Metabolism and Systems Research (IMSR), University of Birmingham, Birmingham, UK.

9 Centre for Endocrinology, Diabetes and Metabolism, Birmingham Health Partners, Birmingham, UK.

${ }^{10}$ Department of Chemical Engineering, Imperial College London, Exhibition Road, London, UK.

\section{Corresponding authors:}

Ben Jones, Section of Endocrinology and Investigative Medicine, Department of Metabolism, Digestion and Reproduction, Imperial College London, London, W12 0NN, United Kingdom. Email: ben.jones@imperial.ac.uk; Telephone: 02033130348

Alejandra Tomas, Section of Cell Biology and Functional Genomics, Department of Metabolism, Digestion and Reproduction, Imperial College London, London, W12 0NN, United Kingdom.

Email: a.tomas-catala@imperial.ac.uk; Telephone: 02075943364

\section{Contents:}

- Supplementary Figure 1

- Supplementary Figure 2

- Supplementary Figure 3

- Supplementary Figure 4

- Supplementary Figure 5 


\section{Supplementary Figure 1}
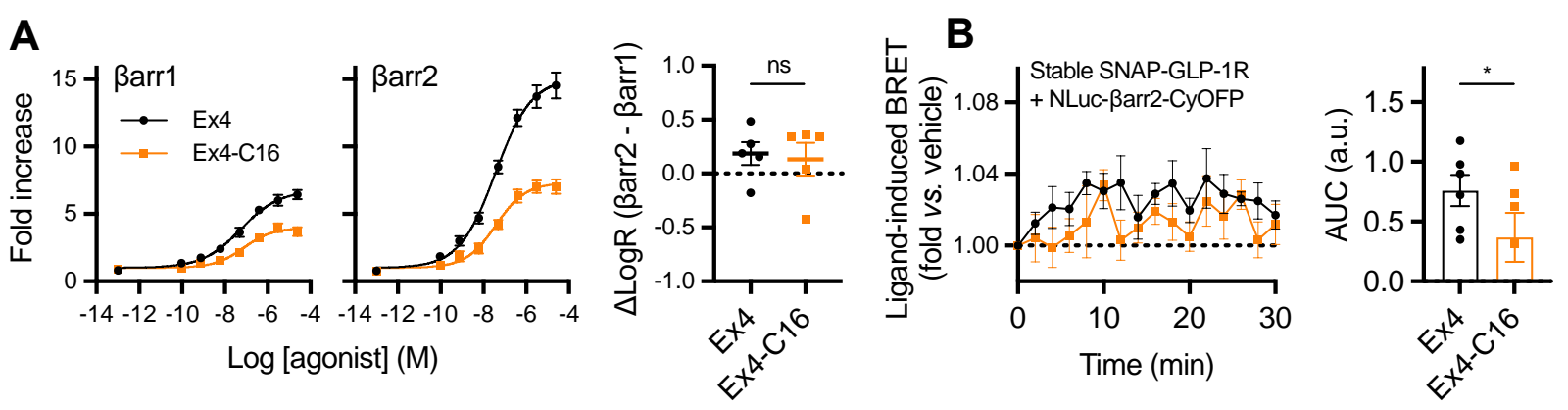

Supplementary Figure 1. Pharmacological responses to exendin-4 and exendin-4-C16. (A) $\beta$-arrestin-1 and $\beta$-arrestin-2 responses measured in PathHunter GLP-1R cells, $n=5$, with bias quantification showing selectivity for $\beta$-arrestin-2 versus $\beta$-arrestin-1 with comparison by paired t-test. (B) Measurement of $\beta$-arrestin-2 activation in HEK293-SNAP-GLP-1R cells transiently transfected with NLuc-4myc-Barr2-CYOFP1 and stimulated with $100 \mathrm{nM}$ agonist or vehicle, $n=6$, with $A U C$ s compared by paired t-test. Data are shown as mean \pm SEM with individual replicates shown for AUC graph. ${ }^{*} p<0.05$ by statistical test indicated. 


\section{Supplementary Figure 2}

A
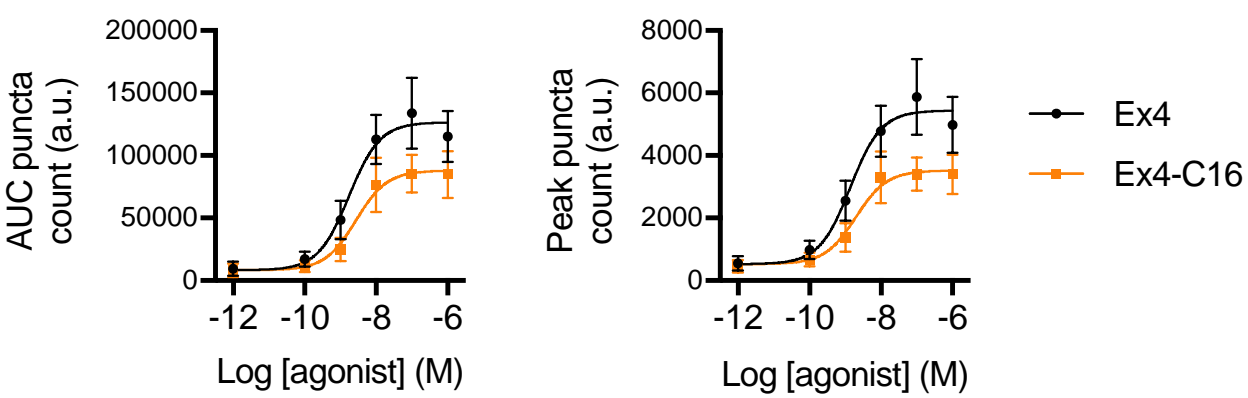

B
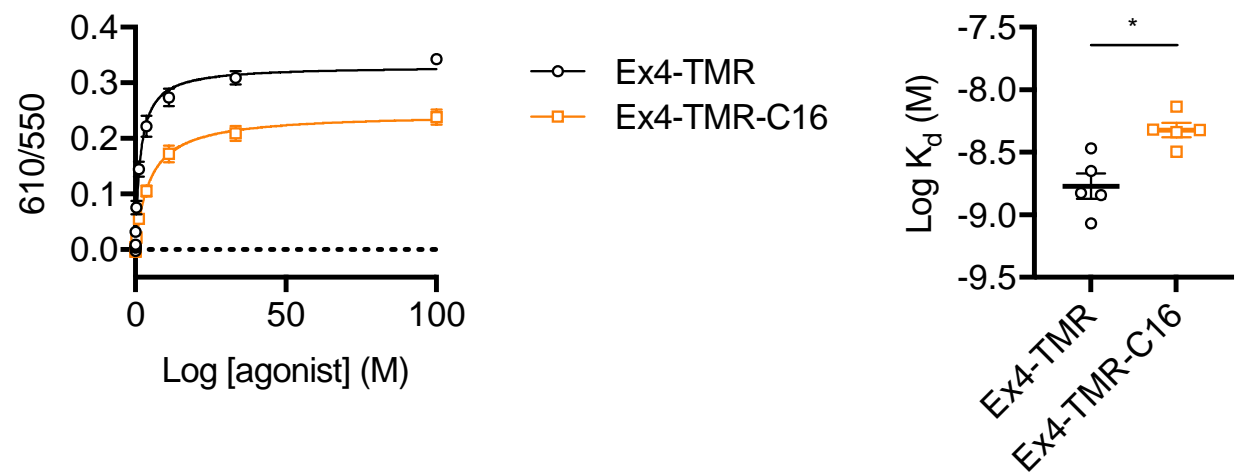

C
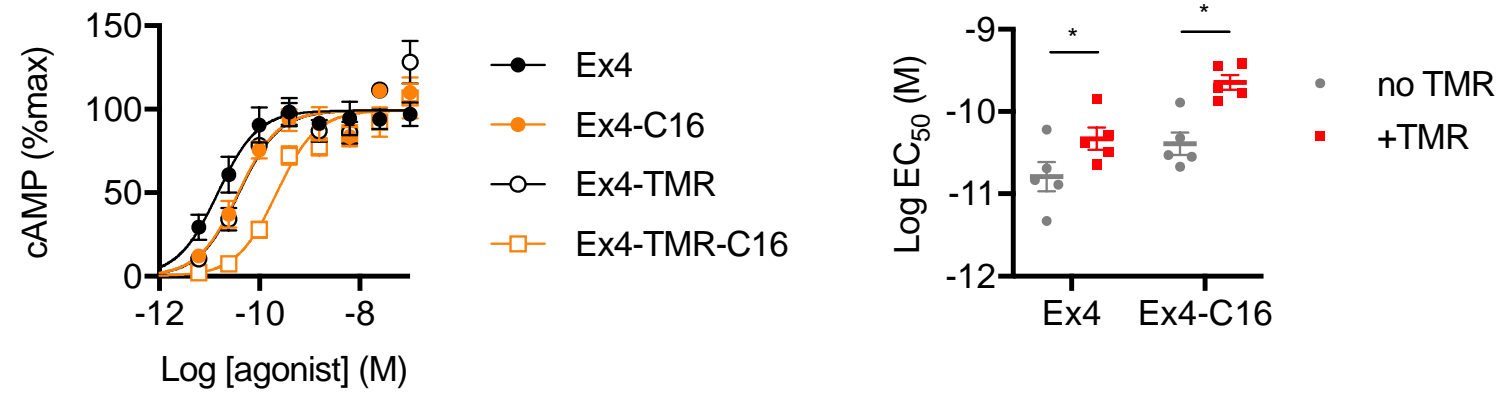

Supplementary Figure 2. Trafficking responses to exendin-4, exendin-4-C16, and functional characterization of TMR-conjugates. (A) Alternative quantification of endosomal puncta formation from Figure 2B, with concentration-dependent peak number of spots, or AUC across the entire 30-min stimulation period indicated with 3-parameter fit shown. (B) TR-FRET binding data for exendin-4-TMR and exendin-4-TMR-C16 in HEK293SNAP-GLP-1R cells, $n=5$, with log $\mathrm{K}_{d}$ compared by paired t-test. (C) cAMP data for TMRmodified or not exendin-4 / exendin-4-C16, $n=5$, with 3-parameter fits shown. LogEC $_{50}$ values are compared by 2 -way randomised block ANOVA with Sidak's test. Data are shown as mean \pm SEM with individual replicates shown where possible. ${ }^{*} p<0.05$ by statistical test indicated 


\section{Supplementary Figure 3}

A

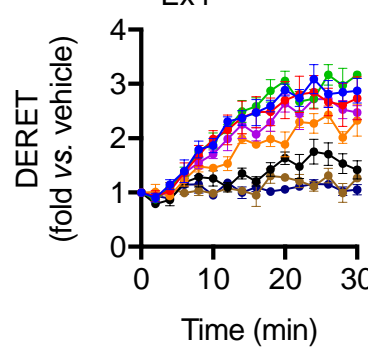

Ex4-C16

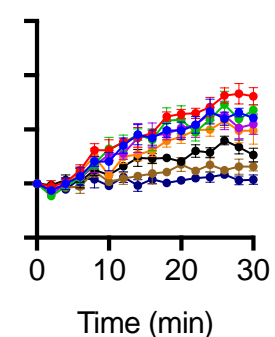

Log [agonist] (M)

$\rightarrow-6.0 \quad \rightarrow-7.9$

$\rightarrow-6.5 \rightarrow-8.4$

$\rightarrow-7.0 \rightarrow-8.9$

$\rightarrow-7.4 \rightarrow$ Veh

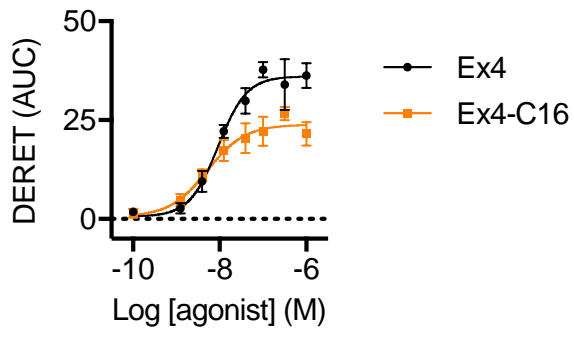

Supplementary Figure 3. GLP-1R trafficking responses by DERET. (A) GLP-1R endocytosis with exendin-4 and exendin-4-C16 measured by DERET in HEK293-SNAPGLP-1R cells, $n=5$. Concentration response is quantified from AUC with 3-parameter fit shown. Data are shown as mean \pm SEM. 


\section{Supplementary Figure 4}

A

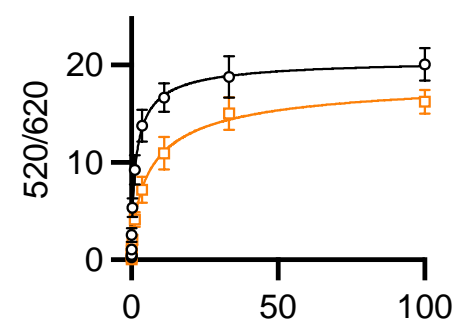

- - Ex4-FITC

$\rightarrow$ Ex4-FITC-C16
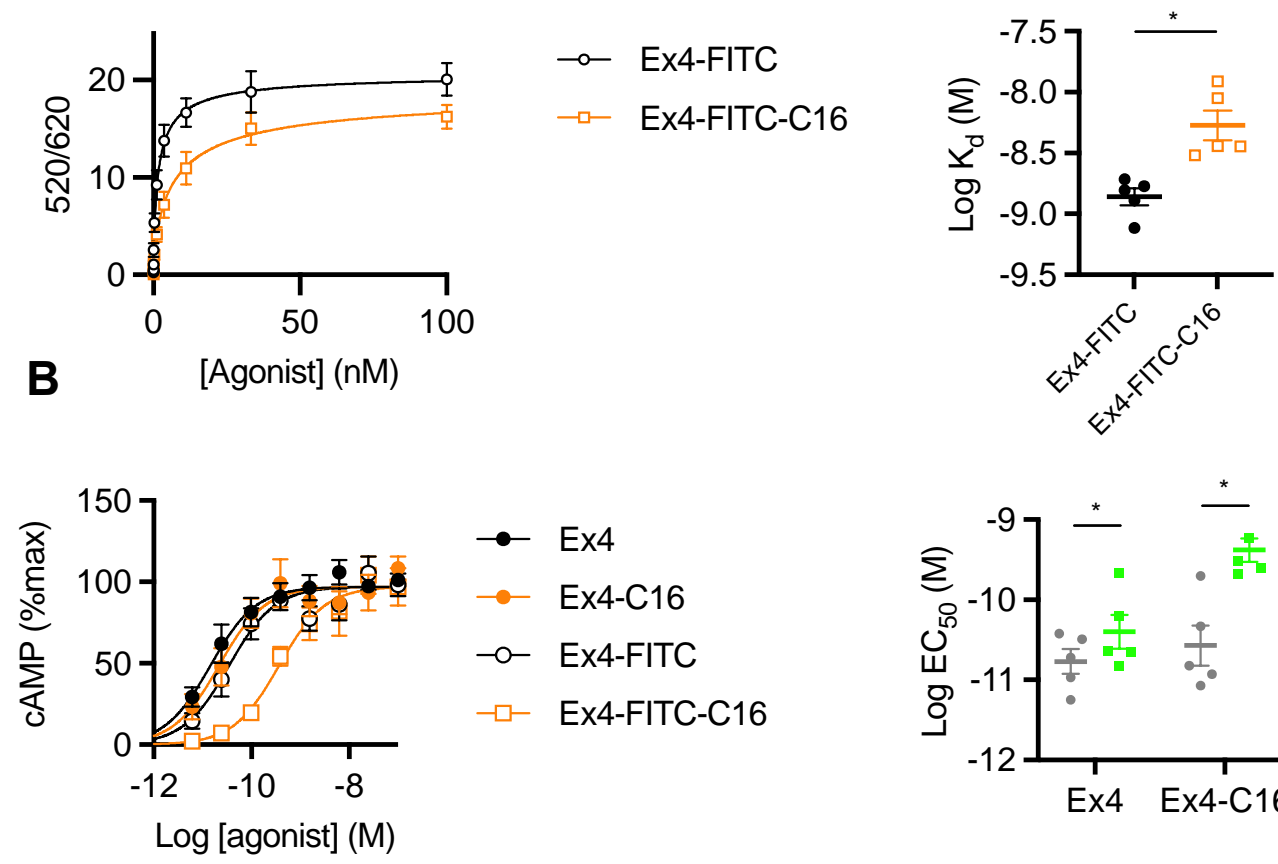

$\rightarrow$ Ex4

$\rightarrow$ Ex4-C16

-o- Ex4-FITC

$\neg-$ Ex4-FITC-C16
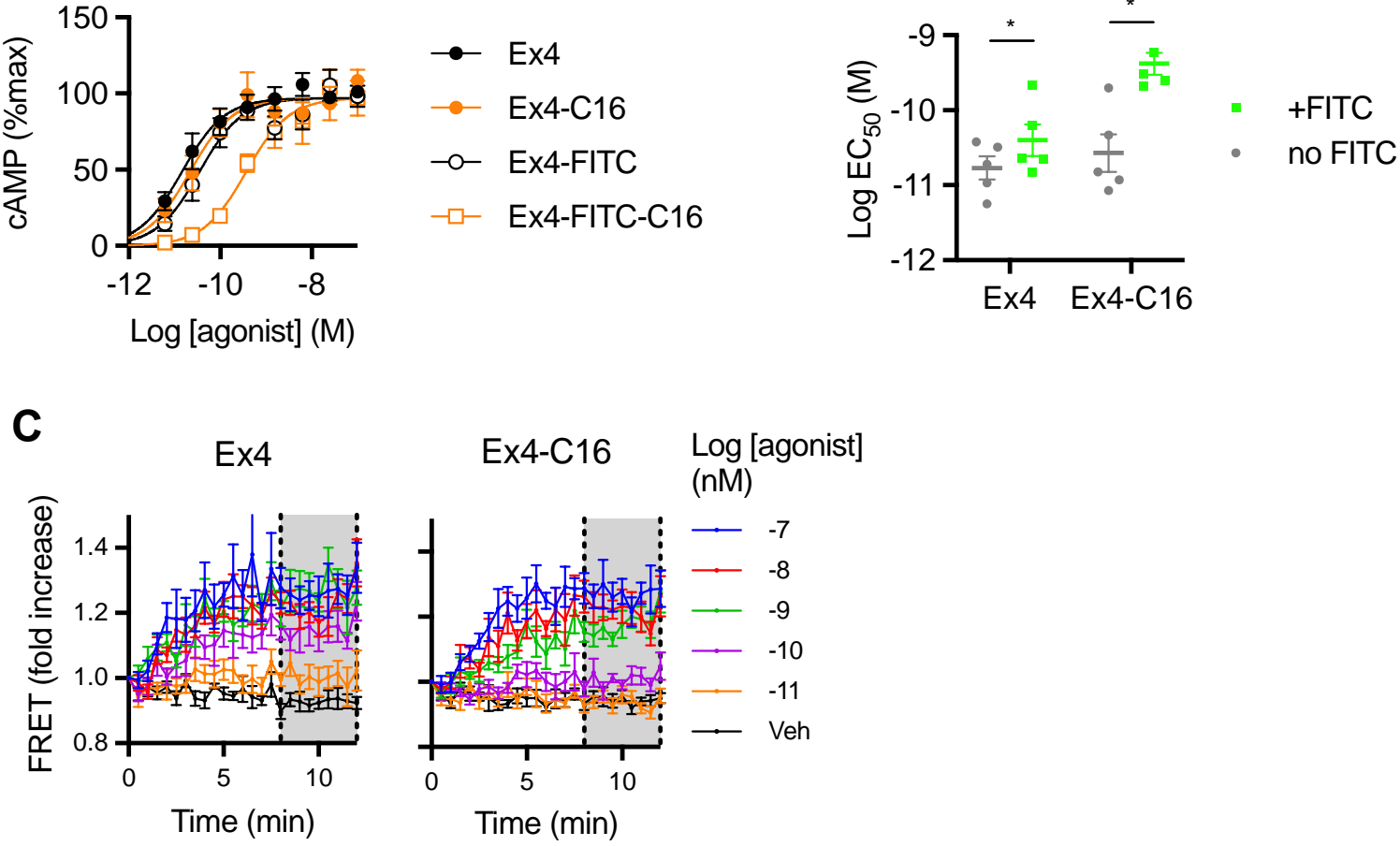

Ex4-C16

Log [agonist]

(nM)

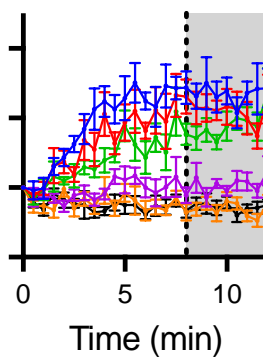

$-7$

$-\quad-8$

- -9

$-\quad-10$

Supplementary Figure 4. Exendin-4-FITC and exendin-4-FITC-C16 functional characterisation. (A) TR-FRET binding data for exendin-4-FITC and exendin-4-FITC-C16 in HEK293-SNAP-GLP-1R cells, $n=5$, with log $K_{d}$ compared by paired t-test. (B) cAMP data for FITC-modified or not exendin-4 / exendin-4-C16, $n=5$, with 3-parameter fits shown. LogEC $_{50}$ values are compared by 2-way randomised block ANOVA with Sidak's test. (C) FRET responses for T-REx-SNAP-GLP-1R cells expressing AKAP79-CUTie stimulated with each ligand, $n=6$. The Shaded area indicates the time-points from which the average response wa calculated in Figure 4D. Data are shown as mean \pm SEM with individual replicates where possible. ${ }^{*} p<0.05$ by statistical test indicated. 


\section{Supplementary Figure 5.}

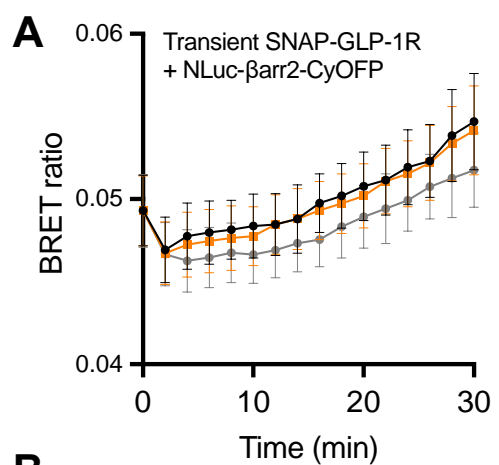

B
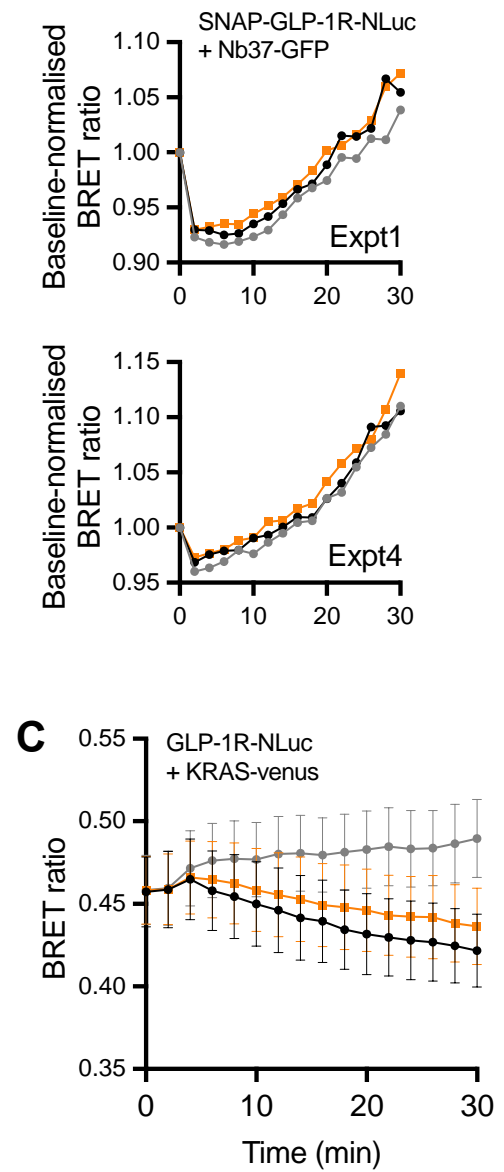

F

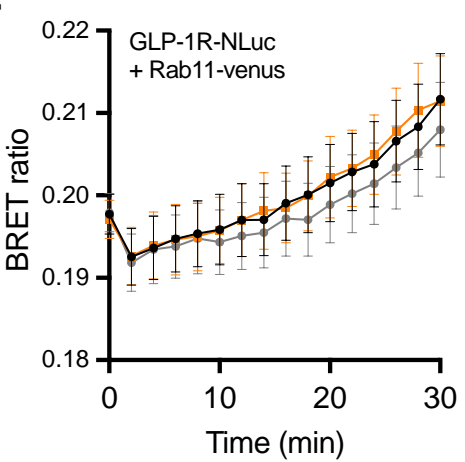

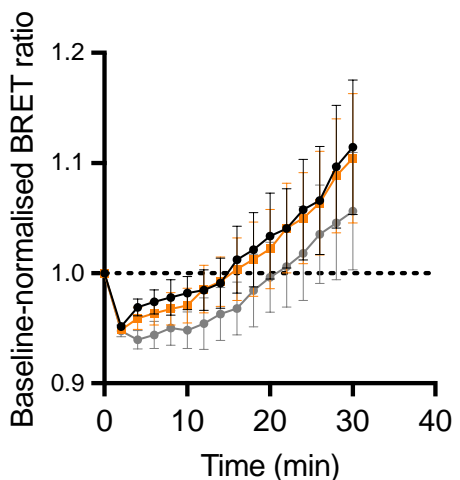
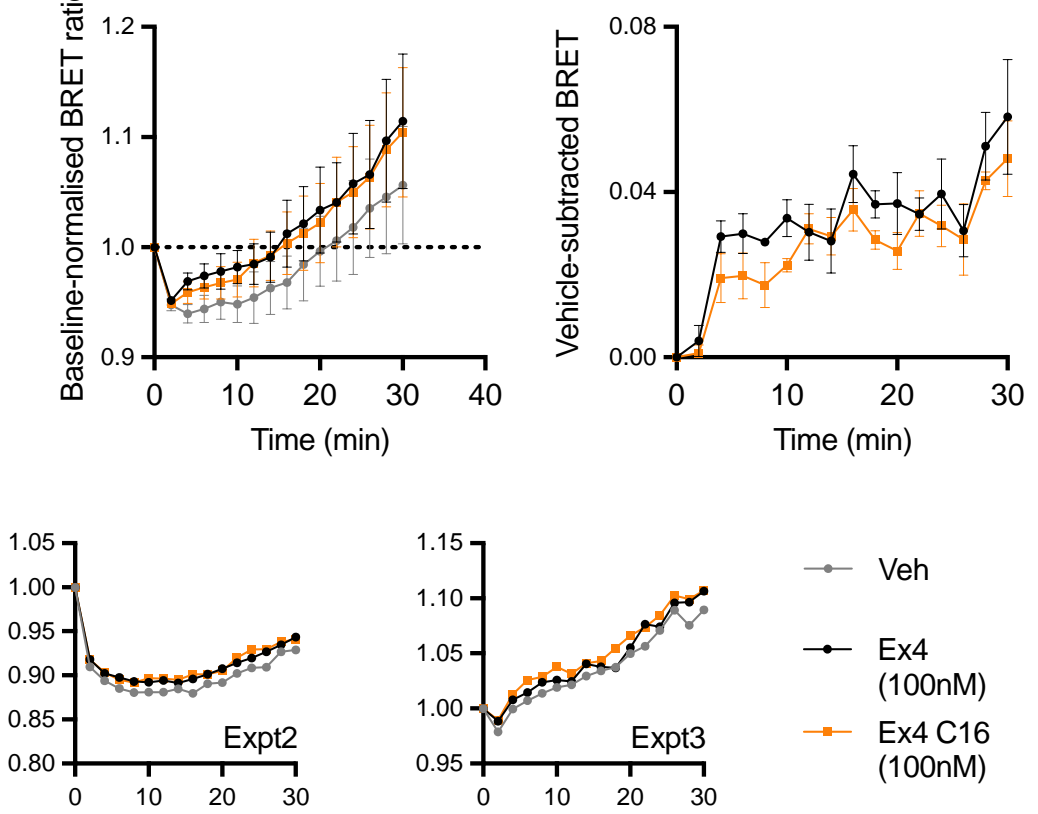

$\because$ Veh

$\rightarrow$ Ex4 (100nM)

- Ex4 C16 (100nM)
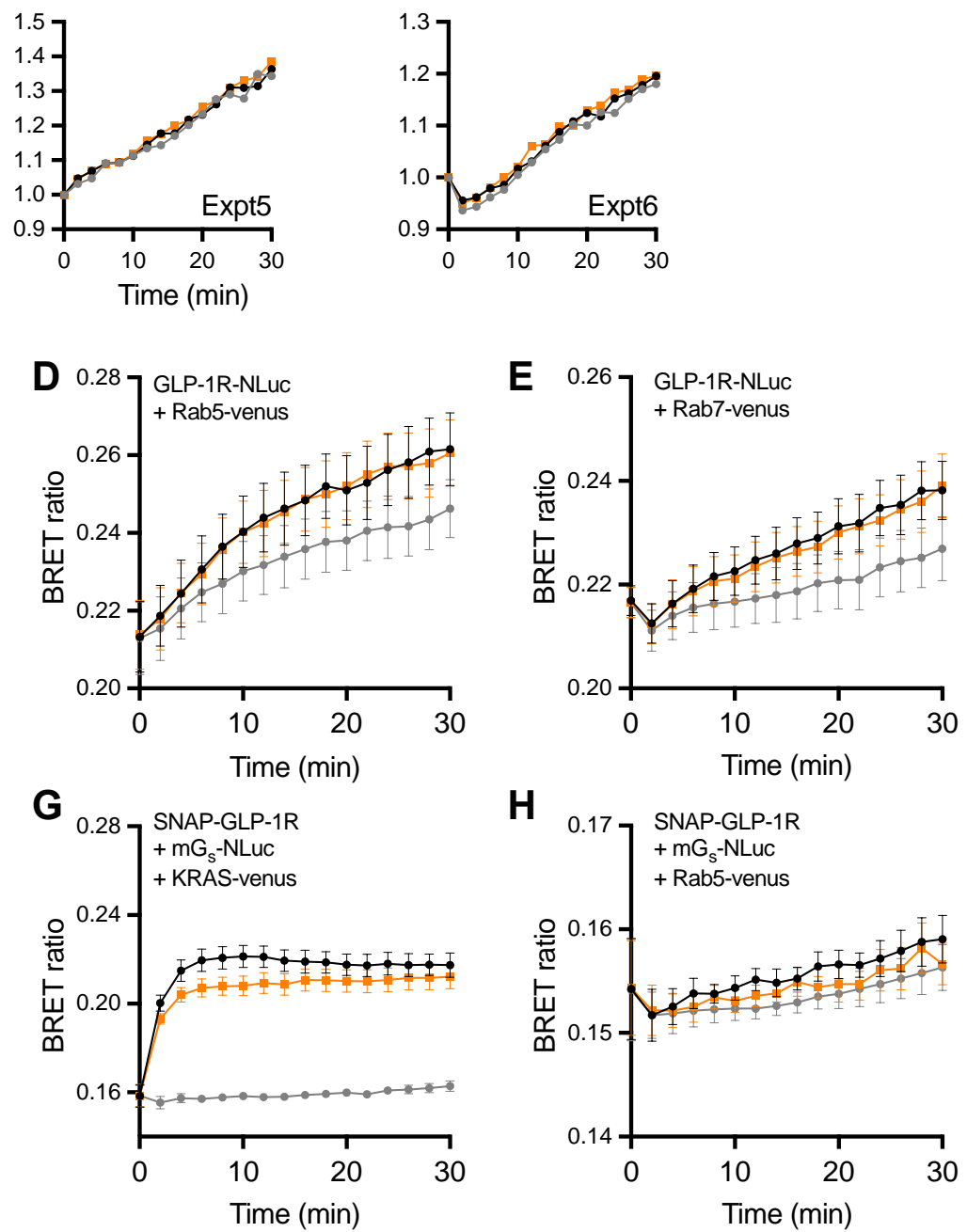
Supplementary Figure 5. BRET analytical approach and raw BRET ratios. (A) $\beta$ arrestin-2 activation assay (refers to Figure 1F). The raw BRET ratios, baseline-normalised, and baseline-normalised and vehicle-subtracted traces are shown to indicate how the specific ligand-induced BRET signal presented in Figure 1F and corresponding AUC values are derived. The BRET signal drift is substantial but agonist-induced BRET ratios are consistently higher at each time-point. (B) Nb37 G protein activation assay (refers to Figure $1 \mathrm{E})$. Due to the low dynamic range of this assay, individual experimental repeats have been presented as baseline-normalised BRET ratios. It can be seen that, in spite of significant drift in all traces over time, agonist-induced BRET values are consistently subtly increased compared to vehicle throughout each experiment. Moreover, on each occasion, exendin-4C16 responses are slightly greater than those of exendin-4. (C), (D), (E) and (F) GLP-1RNluc redistribution assays (refers to Figure $3 F-I)$. (G) and (H) Mini-G $\mathbf{G}_{\mathrm{s}}$ redistribution assay (refers to Figure $3 \mathrm{~K}$ ). Mean \pm SEM is shown. 